\title{
Simulated Comparison Between Different Scenarios of Modular Chicken Breeding Program using MoBPSweb v.1.6.62
}

\section{Wayan Swarautama Mahardhika}

Universitas Gadjah Mada https://orcid.org/0000-0002-3489-3156

Fitriana Nur Laissya Hida

Universitas Gadjah Mada https://orcid.org/0000-0003-2008-1162

Bambang Retnoaji

Universitas Gadjah Mada https://orcid.org/0000-0002-0290-9723

Slamet Widiyanto

Universitas Gadjah Mada https://orcid.org/0000-0002-5877-9112

Torsten Pook

Georg-August-Universität Göttingen https://orcid.org/0000-0001-7874-8500

Budi Setiadi Daryono ( $\square$ bs_daryono@mail.ugm.ac.id)

Universitas Gadjah Mada https://orcid.org/0000-0002-0703-2123

\section{Research Article}

Keywords: chicken breeding, selection scenarios, modular program, stochastic simulation, MoBPSweb

Posted Date: January 25th, 2022

DOl: https://doi.org/10.21203/rs.3.rs-1289983/v2

License: (c) (1) This work is licensed under a Creative Commons Attribution 4.0 International License.

Read Full License 


\section{Simulated Comparison Between Different Scenarios of Modular Chicken Breeding Program using MoBPSweb v.1.6.62}

I Wayan Swarautama Mahardhika ${ }^{1}$, Fitriana Nur Laissya Hida ${ }^{1}$, Bambang Retnoaji ${ }^{2}$, Slamet Widiyanto ${ }^{3}$, Torsten Pook ${ }^{4}$, and Budi Setiadi Daryono, ${ }^{1, *}$

${ }^{1}$ Gama Ayam Research Team, Laboratory of Genetics and Breeding, Faculty of Postgraduate Biology, Universitas Gadjah Mada, 55281 Daerah Istimewa Yogyakarta (DIY), Indonesia

${ }^{2}$ Animal Structure and Development Laboratory, Faculty of Postgraduate Biology, Universitas Gadjah Mada, 55281 Daerah Istimewa Yogyakarta (DIY), Indonesia

${ }^{3}$ Animal Physiology Laboratory, Faculty of Postgraduate Biology, Universitas Gadjah Mada, 55281 Daerah Istimewa Yogyakarta (DIY), Indonesia

${ }^{4}$ Department of Animal Sciences, Animal Breeding and Genetics Group, Center for Integrated Breeding Research, Georg-August-Universität Göttingen, 37075 Göttingen, Germany

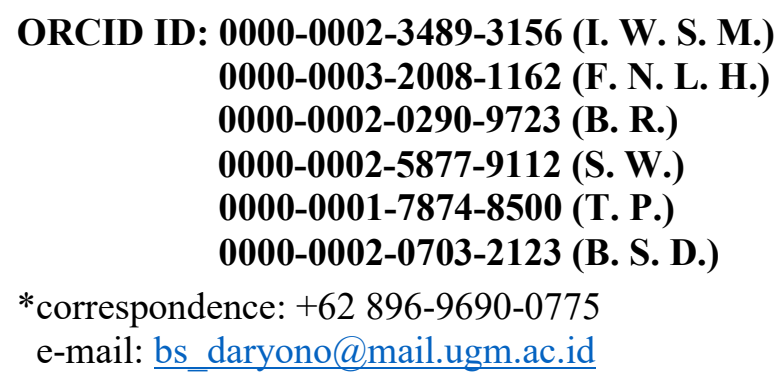

*correspondence: +62 896-9690-0775

e-mail: bs_daryono@mail.ugm.ac.id

(to be submitted 30, 01, 2022)

Background: An optimal selective breeding program must balance ethical risks and operational costs without necessarily compromising its effectiveness and aims. For this purpose, a modular breeding simulator was used. Modular simulation acts as a preliminary evaluation instrument to predict the entire likelihood of outputs from a complex selective breeding program.

Methods: A web-based modular stochastic simulator (MoBPSweb) facilitates the design-test-analysis workflow of the meat-type Pelung chicken selective breeding program with the nickname Gama Ayam Kambro. Several specific selective parameters were formulated and tested against the actual breeding scheme according to Gama Ayam Kambro's research. Three selection scenarios applied to Gama Ayam Kambro's breeding scheme, constructed based on three technical principles of crossings. These scenarios were compared based on accuracy, $F$ coefficient, kinship, and observed phenotypes. An available feature on MoBPSweb also allowed the projections of specific economic parameters.

Results: Crossing techniques, selection model scenarios, and breeding schemes determine the accuracy, $F$ coefficient, kinship, and observed phenotypes. The selection accuracy of male $5^{\text {th }}$ Kambro and Pelung could be optimized using the design of genomic selection scenario_1 and outbreeding crossing techniques, both for Broiler and Pelung selection index. The selection model scenario and similar crossing technique are also quite effective in controlling the $F$ coefficient. However, the outbreeding crossing technique is less effective in increasing the achievements of AFE, BW49D, EN, and FCR, contrarily for FEML, TL, and BW56D. Therefore, for AFE and FEML, the selection of $5^{\text {th }}$ Kambro dan Pelung males could be more optimal by using scenario_2 (BV) and scenario_3 (PHEN), respectively. Meanwhile, for BW49D and EN, the reciprocal applies. Therefore, the selection of $5^{\text {th }}$ Kambro and Pelung males for FCR and BW56D would be more optimal if scenario_1 (GEN) and scenario_3 (PHEN) were used, respectively. Meanwhile, for TL, it would be more optimal if scenario_3 with a phenotype selection design was used for both. The projection of economic parameters indicates that the total operational cost per year is required around \pm 500 million rupiahs for these three selection model scenarios using Gama Ayam Kambro breeding scheme with 100 intensive rearing enclosure units and 50 breeding generations. In addition, the projected operational cost must consider the inflation and interest rate of rupiah per year.

Conclusions: Digitalization of selective breeding program using MoBPSweb stochastic simulator allows the design-test-analysis (DTA) procedure in Gama Ayam breeding scheme and its parameters and scenarios to be executed immediately and the results evaluated in real-time. However, there are at least two things to consider about this research. Firstly, genomic parameters that are specific cause a niche of reference, which means that this research is difficult to compare with other relevant studies. Therefore, the only solution is to do directly testing in the field. Implicitly, it is projected that there will be an exponential increase in the amount of data that must be accommodated by greater computing power to maintain and increase the 
simulation sensitivity. Therefore, these computational requirements may need to be considered one of the operational cost components, especially for the digital integrated poultry industry.

Keywords: chicken breeding; selection scenarios; modular program; stochastic simulation; MoBPSweb

\section{BACKGROUND}

The domestic needs for poultry-based protein sources such as eggs and meats rely heavily on the outsourced products, despite being facilitated locally (Ferlito and Respatiadi 2018). This high dependency may jeopardize domestic and global food security, as Hodson (2017) described. Few may realize that global food security has its limits and can no longer be sustained by conventional means or methods. On the other hand, a growing concern about constant threats from environmental crises and the ever-increasing human population adds to the problem. Although it may seem insignificant, this might mark the beginning of global food security collapse. Gama Ayam Research Team creates a pathway to solving poultry problems in Indonesia. A conceived solution was to tap into the vast biodiversity of Indonesian indigenous chicken breeds. An extensive repertoire of Indonesian indigenous chicken breeds (Nataamijaya 2010; Henuk and Bakti 2018; Mahardhika et al. 2021) provides a unique and promising opportunity to be developed. Developing Indonesian indigenous chicken breeds would help secure food sources domestically, therefore hindering any external interventions or collateral effects caused by the global food crisis.

For years, studies about one Indonesian indigenous chicken breed, Pelung chicken, have been undergone since its conception as the potential candidate of Indonesia-owned meat-type chicken breed by Daryono et al. (2010). During those years, a wide array of research involving selective breeding programs has developed significant key findings regarding its phenotypes and molecular characteristics (Retnoaji et al. 2016; Perdamaian et al. 2017; Utama et al. 2018; Mahardhika and Daryono 2019; Saragih et al. 2019; Mahardhika et al. 2020; Mahardhika et al. 2021; Kurnia et al. 2021; Daryono et al. 2021; Saragih et al. 2021). In conclusion, it was evident that the breed can be further developed to serve as a more sustainable and longterm solution for supporting the local poultry sector. For most of the years, the study mainly revolves around three fundamental aspects: effectiveness, reliability, and minimum ethical cost (Mahardhika et al. 2021). However, the complexity of the selective breeding program could not be handled so easily. The reason was the breeding scheme or genetic architecture and the requisite necessity to create a balance between operational cost, ethical costs, and breeding gains. Thus, it requires a direct experimental or field study and an analytical study, which mainly focuses on predicting the program's outcome.

Collecting data from either internal or other relevant studies outside the Gama Ayam Research Team can be utilized as selective parameters. This library of the selective parameter could serve as a pre-emptive measure to avoid unnecessary chicken sacrifice, unethical rearing, cost requirement, and error probability (Mahardhika et al. 2021). An advanced selective breeding program could be achieved using algorithms to compute these selective parameters into a modular design of a chicken breeding scheme with different scenarios and selections. A preliminary evaluation could be produced without strict parameters and the flexibility to modulate or alternate the breeding scheme or scenarios. This digitalized state would provide an instrument to evaluate, compensate or anticipate any outcome of the selective breeding program with reliable precision and accuracy. The Modular Breeding Program Simulator web version (MoBPSweb) written and constructed by Pook and the team was used to facilitate this purpose. Categorized as stochastic simulation, MoBPS used the R language and was created based on the flaws of its predecessors (Pook et al. 2020; Pook et al. 2021). This study investigated the candidate for meat-type Pelung chicken breed produced from the actual selective breeding program Gama Ayam Kambro (Kampong-Broiler). Different selection scenarios were compared and analyzed using MoBPSweb v.1.6.62. Specific selective parameters were formulated and tested against the breeding scheme according to Gama Ayam Kambro's research. This study also explores the feature provided by MoBPSweb to perform specific economic projections.

\section{METHODS}

\section{Settings and parameters}

The hardware used in this research is an ASUS X450JF-x64 notebook with core processor Intel ${ }^{\circledR}$ Core ${ }^{\mathrm{TM}}$ i7-4700HQ CPU @ 2,4GHz, 2401Mhz, 4 Core(s), 8 Logical Processor(s), and 8 GB RAM capacity. The operating system is Microsoft Windows 10 Education v.10.0.19043.1348 (C) Microsoft Corporation. The observation focuses on the overview Gama Ayam Kambro selective breeding program under three different selection model scenarios. Gama Ayam Kambro's breeding scheme and three selection model scenarios were designed, tested, and analyzed by MoBPSweb v.1.6.62 simulator. The breeding scheme consists of four crossbred generations purebred chicken nucleus as an elder (founder) with the level of crossbreed reaching $5^{\text {th }}$ Filial or $5^{\text {th }}$ Kambro (Figure 1.). Several crossing techniques are used, such as crossbreeding, inbreeding, and outbreeding. The parameter used is constructed based on QTL information from the seven prioritized phenotypic characters in the selection, residual-genetic correlation, selection index, phenotype class, the 
average of phenotype performance, and economic parameters (Supp. File 1, Table 1-4). The seven QTLs were obtained from the ChickenQTLdb chicken QTL database and relevant primer publications. There are seven phenotypic characters, specifically BW49D, BW56D, FEML, AFE, EN, FCR, and TL. Breeding cycle repetition and phenotypic information record (phenotyping) as much as 50 times (generations). BroilerPelung selection index and residual genetic correlation are applied. The designed selection model scenario consist of scenario_1 (Genomic_SD), scenario_2 (Breeding Value_SD), and scenario_3 (Phenotypic_SD). Each one of the selection model scenarios is tested toward the Gama Ayam Kambro breeding scheme. Genomic parameters were constructed using the Ensembl Map Affymetrix Chicken 600K SNPs Array genomic database. The marker density used is $300 \mathrm{~K} \mathrm{SNPs,} \mathrm{with} \mathrm{the} \mathrm{time} \mathrm{unit} \mathrm{simulation} \mathrm{is} \mathrm{set} \mathrm{to} \mathrm{per} \mathrm{week.}$ Additional settings like miraculix and parallel computational are activated. In the genomic selection, the SIM_08 procedure used five core processor with a maximum memory capacity of 30 GB and max the maximum duration of the computational process is 48 hours. The effects of three selection model scenarios on the Gama Ayam Kambro selective breeding program are known according to their output in each chicken nucleus, from the elders to their tillers. The output consists of accuracy, $F$ coefficient, kinship, observed phenotypes, and economic parameter projections related to Gama Ayam Kambro selective breeding program.

\section{Simulation}

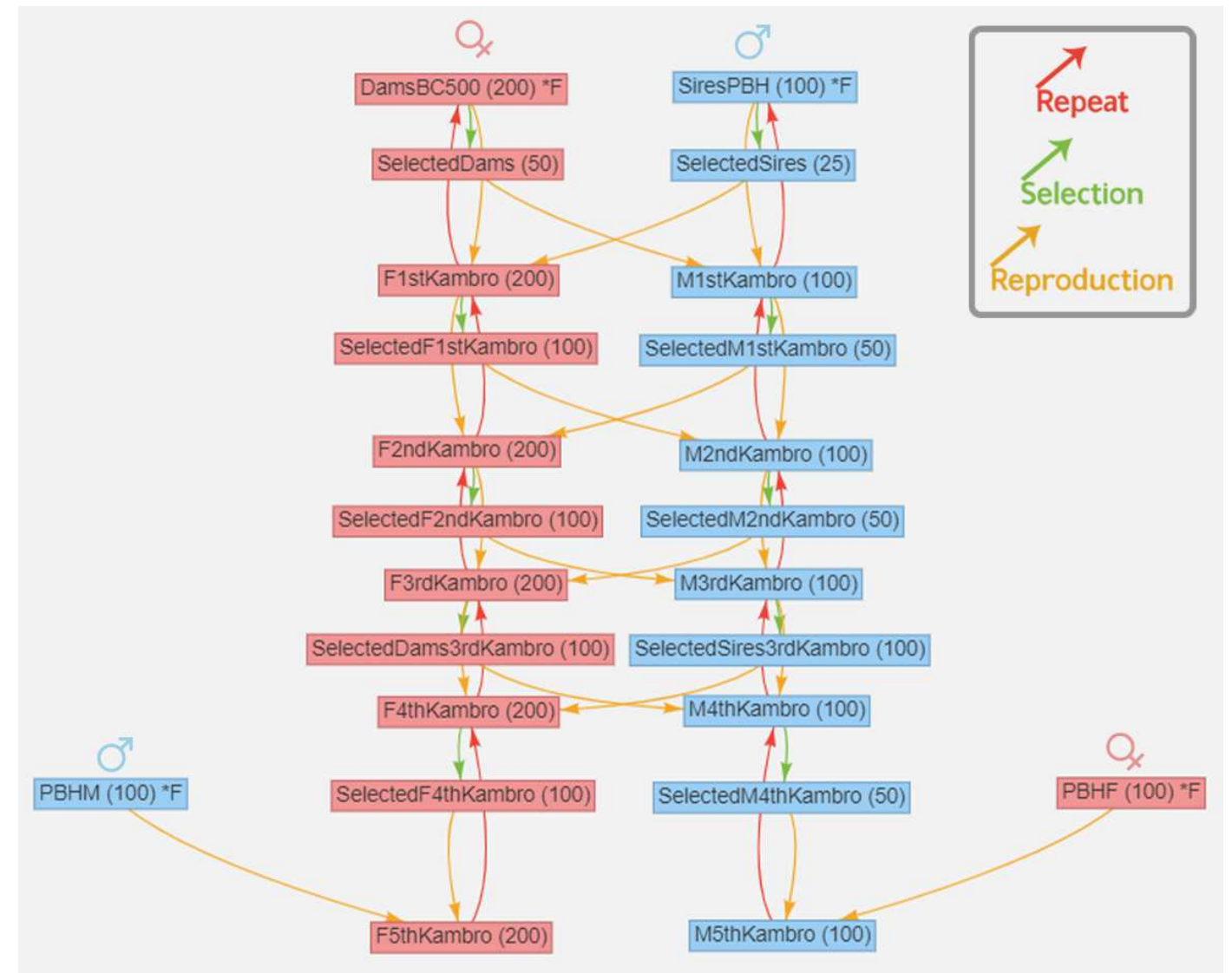

Fig 1. Gama Ayam Kambro breeding scheme. MoBPSweb (2021).

The breeding scheme was designed using nodes (nucleus or cohort) and edges (breeding action) represented by color-coded boxes and arrows in sequence (Figure 1). Boxes of pink represent the chicken group for the hen nucleus $(+$ ) and light blue for the rooster nucleus $(\hat{O})$. Breeding action is represented by red arrows (repeat), green arrows (selection), and orange arrows (reproduction). The repeat code sets the breeding cycles repetition number, selection for selection model scenarios, and reproduction to execute crossbreeding. Gama Ayam Kambro breeding scheme arranged by 200 chicken from BC500 hen nucleus ad 100 chicken from selected and crossed PBH rooster nucleus until it reaches $5^{\text {th }}$ Kambro chicken nucleus. There are three selection model scenarios selected through selection breeding action. Four purebred chicken nuclei arrange Gama Ayam Kambro selective breeding as an elder (founder) with the stage of litters nucleus (progeny) until it reaches the $5^{\text {th }}$ Filial $\left(\mathrm{F}_{5}\right)$ or $5^{\text {th }}$ Kambro (Figure 1). The purebred elders chicken nucleus comprises one hen nucleus, two PBH roosters nucleus, and one BC500 hen nucleus. Each individual will be selected and crossed via reproduction breeding action. The total of generations or breeding cycles repetition is determined by repeat breeding action. Selection model scenarios in the Gama Ayam Kambro scheme are constructed based on scenario_1 (Genomic_SD), scenario_2 (Breeding Value_SD), and scenario_3 (Phenotypic_SD). 
Step 1. + BC500 nucleus as [200 chickens], type of genotype generation [whole homozygote], phenotype class [whole phenotyped], and operational cost [chicken feed, vaccine, and supplement]. Selected nucleus of 9 BC500 [50 chickens], duration [52 weeks], selection type [BVE], BVE methods [DMM], selection index [Broiler], selection proportion [0.25], kinship matrix [VanRaden], and nucleus for BVE [manual selection of two prevous repetitive]. Step 2. $\delta$ PBH nucleus [100 chickens], type of genotype generation [random sample], phenotype class [whole phenotyped], and operational cost [chicken feed, vaccine, and supplement]. Selected nucleus of $\lesssim$ PBH [25 chickens], duration [52 weeks], selection type [PHEN], selection index [Pelung], and selection proportion [0.25]. Step 3. The selected nucleus of $q$ BC500 is crossed with the nucleus of selected $\widehat{\partial}$ PBH with $[50$ times] breeding cycles. That crossbreed produces $+1^{\text {st }}$ Kambro and $\delta^{\lambda} 1^{\text {st }}$ Kambro nucleus.

Step 4. The nucleus of $q 1^{\text {st }}$ Kambro [200 chickens], phenotype class [whole phenotyped], and operational cost [chicken feed, vaccine, and supplement]. Selected nucleus of $1^{\text {st }}$ Kambro [100 chickens], ], duration [52 weeks], selection type [BVE], BVE methods [REML-(G)BLUP], selection index [Broiler], selection proportion [0.5], kinship matrix [VanRaden], and nucleus for BVE [manual selection of two prevous repetitive].

Step 5. The nucleus of $\widehat{\sigma} 1^{\text {st }}$ Kambro [100 chickens], phenotype class [whole phenotyped], and operational cost [chicken feed, vaccine, and supplement]. Selected nucleus of $\partial^{\Uparrow} 1^{\text {st }}$ Kambro [50 chickens], duration [52 weeks], selection type [BVE], BVE methods [REML-(G)BLUP], index selection [Pelung], proportion index [0.5], kinship matrix [VanRaden], and nucleus for BVE [manual selection of two prevous repetitive].

Step 6. The nucleus of $q 1^{\text {st }}$ Kambro is crossed with selected $\delta^{\Uparrow} 1^{\text {st }}$ Kambro [50 times] breeding cycles. Those crossbreeding produce the nucleus of $2^{\text {nd }}$ Kambro and $\delta 2^{\text {nd }}$ Kambro.

Step 7. The nucleus of $q 2^{\text {nd }}$ Kambro [200 chickens], phenotype class [whole phenotyped], and operational cost [chicken feed, vaccine, and supplement]. Selected nucleus of $q 2^{\text {nd }}$ Kambro [100 chickens], duration [52 weeks], selection type [BVE], BVE method [REML-(G)BLUP], selection index [Broiler], selection proportion [0.5], kinship matrix [VanRaden], and nucleus for BVE [manual selection of two prevous repetitive].

Step 8. The nucleus of $\lesssim 2^{\text {nd }}$ Kambro [100 chickens], phenotype class [whole phenotyped], and operational cost [chicken feed, vaccine, and supplement]. Selected nucleus of $2^{\text {nd }}$ Kambro [50 chickens], duration [52 week], selection type [BVE], BVE method [REML-(G)BLUP], selection index [Pelung], selection proportion [0.5], kinship matrix [VanRaden], and nucleus for BVE [manual selection of two previous repetitive].

Step 9. The selected nucleus of $q 2^{\text {nd }}$ Kambro is crossed with the nucleus of selected $\delta 2^{\text {nd }}$ Kambro with a [50 times] breeding cycle. The crossing breed produces a nucleus of $q 3^{\text {rd }}$ Kambro and $\delta^{\lambda} 3^{\text {rd }}$ Kambro chickens.

Step 10. The nucleus of $q 3^{\text {rd }}$ Kambro as much as [200 chickens], phenotype class [whole phenotype], and operational costs [chicken feed, vaccines, and supplements]. Nucleus of selected $\odot 3^{\text {rd }}$ Kambro as much as [100 chickens], duration [52 weeks], selection type [BVE], BVE method [REML-(G)BLUP], selection index [Broiler], selection proportion [1], kinship matrix [VanRaden], and nucleus for BVE [manual selection of two previous replications].

Step 11. The nucleus of $\lesssim 3^{\text {rd }}$ Kambro as much as [100 chickens], phenotype class [whole phenotyped], and operational costs [chicken feed, vaccines, and supplements]. Selected nucleus of $q 3^{\text {rd }}$ Kambro as much [50 chickens], duration [52 weeks], selection type [BVE], BVE method [REML-(G)BLUP], selection index [Pelung], selection proportion [1], kinship matrix [VanRaden], and nucleus for BVE [manual selection of two previous replications].

Step 12. The nucleus of selected $q 3^{\text {rd }}$ Kambro is crossed with the selected nucleus of $\jmath^{\text {rd }}$ Kambro with a breeding cycle of [50 times]. That crossbreed produces the nucleus of $q 4^{\text {th }}$ Kambro dan $\delta 4^{\text {th }}$ Kambro.

Step 13. The nucleus of $+4^{\text {th }}$ Kambro as much as [ 200 chickens], phenotype class [whole phenotyped], and operational costs [chicken feed, vaccines, and supplements]. Selected nucleus of $q 4^{\text {th }}$ Kambro as much as [100 chickens], duration [52 weeks], selection type [BVE], BVE method [REML-(G)BLUP], selection index [Broiler], selection proportion [0.5], kinship matrix [VanRaden], and nucleus for BVE [manual selection of two previous replications].

Step 14. The nucleus of $\lesssim 4^{\text {th }}$ Kambro as much as [100 chickens], phenotype class [whole phenotyped], and operational costs [chicken feed, vaccines, and supplements]. Selected nucleus of ${ }^{\lambda} 4^{\text {th }}$ Kambro [50 chickens], duration [52 weeks], selection type [BVE], BVE method [REML-(G)BLUP], selection index [Pelung], selection proportion [0.5], kinship matrix [VanRaden], and nucleus for BVE [manual selection of two previous replications]. Step 15. The selected nucleus of $\widehat{\jmath} \mathrm{PBH}$ as much as [100 chickens], genotype generation type [fully A allele], phenotype class [whole phenotype], and operational costs [chicken feed, vaccines, and supplements]. The selected nucleus of $q 4^{\text {th }}$ Kambro is crossed with the nucleus of ${ }^{\top}$ PBH with a breeding cycle of [50 times]. The crossbreed produces a nucleus of $q 5^{\text {th }}$ Kambro chickens.

Step 16. The nucleus of $q \mathrm{PBH}$ as much as [100 chickens], genotype generation type [fully A allele], phenotype class [whole phenotype], and operational costs [chicken feed, vaccines, and supplements]. The selected nucleus of $\lambda$ $4^{\text {th }}$ Kambro is crossed with the nucleus of $O \mathrm{PBH}$ with a breeding cycle of [50 times]. The crossbreed produces a nucleus of $\delta^{\text {th }}$ Kambro chickens.

\section{Scenario_2 (Breeding Value_SD)}

Step 1. The nucleus of $q$ BC500 as much as [200 chickens], genotype generation type [whole homozygote], phenotype class [whole phenotyped], and operational costs [chicken feed, vaccines, and supplements]. Selected nucleus of + BC500 as much as [50 chickens], duration [52 weeks], selection type [BVE], BVE method [DMM], 
selection index [Broiler], selection proportion [0.25], kinship matrix [VanRaden], and nucleus for BVE [manual selection of two previous replications].

Step 2. The nucleus of $\widehat{\partial} \mathrm{PBH}$ as many as [100 chickens], genotype generation type [full heterozygotes], phenotype class [whole phenotyped], and operational costs [chicken feed, vaccines, and supplements]. Selected nucleus of $\lambda$ PBH as many as [25 chickens], duration [52 weeks], selection type [BVE], BVE method [DMM], selection index [Pelung], selection proportion [0.25], kinship matrix [pedigree], pedigree depth [7 levels], and nucleus for BVE [manual selection of two previous tests].

Step 3. The selected nucleus of $q$ BC500 is crossed breeding with a selected nucleus of $\widehat{\jmath} \mathrm{PBH}$ with a breeding cycle [50 times]. The crossbreed produces the nucleus of $\bigcirc 1^{\text {st }}$ Kambro and $\delta 1^{\text {st }}$ Kambro chickens.

Step 4. The nucleus of $q 1^{\text {st }}$ Kambro as many as [200 chickens], phenotype class [whole phenotyped], and operational costs [chicken feed, vaccines, and supplements]. Selected nucleus of $q 1^{\text {st }}$ Kambro as many as [100 chickens], duration [52 weeks], selection type [BVE], BVE method [REML-(G)BLUP], selection index [Broiler], selection proportion [0.5], kinship matrix [VanRaden], and nucleus for BVE [manual selection of two previous replications].

Step 5. The nucleus of $\widehat{\delta} 1^{\text {st }}$ Kambro as many as [100 chickens], phenotype class [whole phenotyped], and operational costs [chicken feed, vaccines, and supplements]. Selected nucleus of $\delta^{\lambda} 1^{\text {st }}$ Kambro as many as [50 chickens], duration [52 weeks], selection type [BVE], BVE method [REML-(G)BLUP], selection index [Pelung], selection proportion [0.5], kinship matrix [VanRaden], and nucleus for BVE [manual selection of two previous replications].

Step 6. The selected nucleus of $q 1^{\text {st }}$ Kambro is crossed breeding with a selected nucleus of $\delta^{\lambda} 1^{\text {st }}$ Kambro with breeding cycle [50 times]. The crossbreed produces the nucleus of $q 2^{\text {nd }}$ Kambro and $\widehat{\delta} 2^{\text {nd }}$ Kambro chickens. Step 7. The nucleus of $q 2^{\text {nd }}$ Kambro as many as [200 chickens], phenotype class [whole phenotyped], and operational costs [chicken feed, vaccines, and supplements]. Selected nucleus of $q 2^{\text {nd }}$ Kambro as many as [100 chickens], duration [52 weeks], selection type [BVE], BVE method [REML-(G)BLUP], selection index [Broiler], selection proportion [0.5], kinship matrix [VanRaden], and nucleus for BVE [manual selection of two previous replications].

Step 8. The nucleus of $\hat{\partial} 2^{\text {nd }}$ Kambro as many as [100 chickens], phenotype class [whole phenotyped], and operational costs [chicken feed, vaccines, and supplements]. Selected nucleus of $\sigma^{-1} 2^{\text {nd }}$ Kambro as many as [50 chickens], duration [52 weeks], selection type [BVE], BVE method [REML-(G)BLUP], selection index [Pelung], selection proportion [0.5], kinship matrix [VanRaden], and nucleus for BVE [manual selection of two previous replications].

Step 9. The selected nucleus $q 2^{\text {nd }}$ Kambro is crossed breeding with a selected nucleus of $\sigma^{\lambda} 2^{\text {nd }}$ Kambro with a breeding cycle [50 times]. The crossbreed produces the nucleus of $q 3^{\text {rd }}$ Kambro and $\delta^{\lambda} 3^{\text {rd }}$ Kambro chickens. Step 10. The nucleus of $q 3^{\text {rd }}$ Kambro as many as [200 chickens], phenotype class [whole phenotyped], and operational costs [chicken feed, vaccines, and supplements]. Selected nucleus of $q 3^{\text {rd }}$ Kambro as many as [100 chickens], duration [52 weeks], selection type [BVE], BVE method [REML-(G)BLUP], selection index [Broiler], selection proportion [0.5], kinship matrix [VanRaden], and nucleus for BVE [manual selection of two previous replications].

Step 11. The nucleus of $\widehat{\diamond} 3^{\text {rd }}$ Kambro as many as [100 chickens], phenotype class [whole phenotyped], and operational costs [chicken feed, vaccines, and supplements]. Selected nucleus of $\delta^{7} 3^{\text {rd }}$ Kambro as many as [50 chickens], duration [52 weeks], selection type [BVE], BVE method [REML-(G)BLUP], selection index [Pelung], selection proportion [1], kinship matrix [VanRaden], and nucleus for BVE [manual selection of two previous replications].

Step 12. The selected nucleus $q 3^{\text {rd }}$ Kambro is crossed breeding with a selected nucleus of $\oint^{\lambda} 3^{\text {rd }}$ Kambro with a breeding cycle [50 times]. The crossbreed produces the nucleus of $q 4^{\text {th }}$ Kambro and $\delta 4^{\text {th }}$ Kambro chickens. Step 13. The nucleus of $O 4^{\text {th }}$ Kambro as many as [200 chickens], phenotype class [whole phenotyped], and operational costs [chicken feed, vaccines, and supplements]. Selected nucleus of $q 4^{\text {th }}$ Kambro as many as [100 chickens], duration [52 weeks], selection type [BVE], BVE method [REML-(G)BLUP], selection index [Broiler], selection proportion [0.5], kinship matrix [VanRaden], and nucleus for BVE [manual selection of two previous replications].

Step 14. The nucleus of $\lesssim 4^{\text {th }}$ Kambro as many as [100 chickens], phenotype class [whole phenotyped], and operational costs [chicken feed, vaccines, and supplements]. Selected nucleus of $\delta^{\text {th }} 4^{\text {th }}$ Kambro as many as [50 chickens], duration [52 weeks], selection type [BVE], BVE method [REML-(G)BLUP], selection index [Pelung], selection proportion [0.5], kinship matrix [VanRaden], and nucleus for BVE [manual selection of two previous replications].

Step 15. The nucleus of $\widehat{\jmath}$ PBH as many as [100 chickens], generation genotype type [random sample], phenotype class [whole phenotyped], and operational costs [chicken feed, vaccines, and supplements]. Selected nucleus $q^{\text {th }}$ Kambro is a crossbreed with the nucleus of $\widehat{\curvearrowright} \mathrm{PBH}$ with a breeding cycle [50 times]. That crossed breed produces the nucleus of $q 5^{\text {th }}$ Kambro as many as [200].

Step 16. The nucleus of $q \mathrm{PBH}$ as many as [100 chickens], genotype generation type [random sample], phenotype class [whole phenotyped class], and operational costs [chicken feed, vaccines, and supplements]. The selected nucleus of $\delta^{\text {th }}$ Kambro is a crossbreed with the nucleus of + PBH with a breeding cycle as [50 times]. That crossed breed produces a nucleus of $\delta^{\text {th }}$ Kambro as many as [100]. 
Step 1. The nucleus of $q$ BC500 as many as [200 chickens], genotype generation type [fully A allele], phenotype class [whole phenotyped], and operational costs [chicken feed, vaccines, and supplements].

Selected nucleus of $q$ BC500 as many as [50 chickens], duration [52 weeks], selection type [BVE], BVE method [DMM], selection index [Broiler], selection proportion [0.25], kinship matrix [pedigree], and nucleus for BVE [manual selection of two previous replications].

Step 2. The nucleus of $\hat{\partial} \mathrm{PBH}$ as many as [100 chickens], genotype generation type [random sample], phenotype class [whole phenotyped], and operational costs [chicken feed, vaccines, and supplements]. Selected nucleus of $\sigma^{\lambda}$ $\mathrm{PBH}$ as many as [25 chickens], duration [52 weeks], selection type [PHEN], selection index [Pelung], and selection proportion [0.25].

Step 3. The selected nucleus of $q$ BC500 is crossed breeding with a selected nucleus of $\delta$ PBH with a breeding cycle [50 times]. That crossbreed produces the nucleus of $q^{\text {st }}$ Kambro and $\delta^{\wedge} 1^{\text {st }}$ Kambro chickens.

Step 4. The nucleus of $q 1^{\text {st }}$ Kambro as many as [200 chickens], phenotype class [whole phenotyped], and operational costs [chicken feed, vaccines, and supplements]. Selected nucleus of $q 1^{\text {st }}$ Kambro as many as [100 chickens], duration [52 weeks], selection type [PHEN], selection index [Broiler], and selection proportion [0.5]. Step 5. The nucleus of $\hat{\delta} 1^{\text {st }}$ Kambro as many as [100 chickens], phenotype class [whole phenotyped], and operational costs [chicken feed, vaccines, and supplements]. Selected nucleus of $\delta 1^{\text {st }}$ Kambro as many as [50 chickens], duration [52 weeks], selection type [PHEN], selection index [Pelung], and selection proportion [0.5]. Step 6. The selected nucleus of $\bigcirc 1^{\text {st }}$ Kambro is crossed breeding with a selected nucleus of $\delta^{1 \text { st }}$ Kambro with breeding cycle [50 times]. The crossbreed produces the nucleus of $q 2^{\text {nd }}$ Kambro and $\delta 2^{\text {nd }}$ Kambro chickens.

Step 7. The nucleus of $q 2^{\text {nd }}$ Kambro as many as [200 chickens], phenotype class [whole phenotyped], and operational costs [chicken feed, vaccines, and supplements]. Selected nucleus of $q 2^{\text {nd }}$ Kambro as many as [100 chickens], duration [52 weeks], selection type [PHEN], selection index [Broiler], and selection proportion [0.5]. Step 8. The nucleus of $\hat{\partial} 2^{\text {nd }}$ Kambro as many as [100 chickens], phenotype class [whole phenotyped], and operational costs [chicken feed, vaccines, and supplements]. Selected nucleus of $\delta^{\lambda} 2^{\text {nd }}$ Kambro as many as [50 chickens], duration [52 weeks], selection type [PHEN], selection index [Pelung], and selection proportion [0.5]. Step 9. The selected nucleus $q 2^{\text {nd }}$ Kambro is crossed breeding with a selected nucleus of $\widehat{o} 2^{\text {nd }}$ Kambro with a breeding cycle [50 times]. The crossbreed produces the nucleus of $q 3^{\text {rd }}$ Kambro and $\delta^{\lambda} 3^{\text {rd }}$ Kambro chickens.

Step 10. The nucleus of $q 3^{\text {rd }}$ Kambro as many as [200 chickens], phenotype class [whole phenotyped], and operational costs [chicken feed, vaccines, and supplements]. Selected nucleus of $\odot 3^{\text {rd }}$ Kambro as many as [100 chickens], duration [52 weeks], selection type [PHEN], selection index [Broiler], and selection proportion [0.5]. Step 11. The nucleus of $\widehat{\partial} 3^{\text {rd }}$ Kambro as many as [100 chickens], phenotype class [whole phenotyped], and operational costs [chicken feed, vaccines, and supplements]. Selected nucleus of $\delta^{2} 3^{\text {rd }}$ Kambro as many as [50 chickens], duration [52 weeks], selection type [PHEN], selection index [Pelung], and selection proportion [1]. Step 12. The selected nucleus $q 3^{\text {rd }}$ Kambro is crossed breeding with a selected nucleus of $\delta^{\lambda} 3^{\text {rd }}$ Kambro with a breeding cycle [50 times]. The crossbreed produces the nucleus of $q 4^{\text {th }}$ Kambro and $\delta^{\lambda} 4^{\text {th }}$ Kambro chickens.

Step 13. The nucleus of $q 4^{\text {th }}$ Kambro as many as [200 chickens], phenotype class [whole phenotyped], and operational costs [chicken feed, vaccines, and supplements]. Selected nucleus of $\varphi_{4}^{\text {th }}$ Kambro as many as [100 chickens], duration [52 weeks], selection type [PHEN], selection index [Broiler], and selection proportion [0.5]. Step 14. The nucleus of $\delta^{\lambda} 4^{\text {th }}$ Kambro as many as [100 chickens], phenotype class [whole phenotyped], and operational costs [chicken feed, vaccines, and supplements]. Selected nucleus of $\sigma^{2} 4^{\text {th }}$ Kambro as many as [50 chickens], duration [52 weeks], selection type [PHEN], selection index [Pelung], and selection proportion [0.5]. Step 15. The nucleus of $\hat{\mathrm{PBH}}$ as many as [100 chickens], generation genotype type [random sample], phenotype class [whole phenotyped], and operational costs [chicken feed, vaccines, and supplements]. Selected nucleus $q 4^{\text {th }}$ Kambro is a crossbreed with the nucleus of $\delta \mathrm{PBH}$ with a breeding cycle [50 times]. That crossed breed produces the nucleus of $q 5^{\text {th }}$ Kambro.

Step 16. The nucleus of $\odot \mathrm{PBH}$ as many as [100 chickens], genotype generation type [random sample], phenotype class [whole phenotyped class], and operational costs [chicken feed, vaccines, and supplements]. The selected nucleus of $\sigma^{\lambda} 4^{\text {th }}$ Kambro is a crossbreed with the nucleus of $O \mathrm{PBH}$ with a breeding cycle as [50 times]. That crossed breed produces a nucleus of $\delta 5^{\text {th }}$ Kambro.

\section{RESULTS \& DISCUSSION}

A review of accuracy from three selection model scenarios in the Gama Ayam Kambro selective breeding program with 50 generations are elaborated per chicken nucleus for each selection index, from the elders to their litters (Figure 2). For the Broiler selection index, in the nucleus of selected $\bigcirc$ BC500 and $\sigma^{\lambda}$ PBH elderly chicken, the three selection model scenarios have the upper and lower accuracy limit of $95 \%$ and $60 \%$, respectively, with the upper and lower limit of accuracy extreme fluctuations in each generation. In the $q / O 1^{\text {st }}$ and $2^{\text {nd }}$ Kambro chicken nucleus, there is still fluctuation followed by an increase in the accuracy's lower limit to $70 \%$. Then, stability begins to be achieved in the $9 / \delta^{\text {rd }}$ and $4^{\text {th }}$ Kambro chicken nucleus, followed by a decrease in the accuracy's upper limit to $80 \%$. In the $\$ / \widehat{\Im} 3^{\text {rd }}$ and $4^{\text {th }}$ Kambro chicken nucleus, an extreme lower limit of accuracy by $-20 \%$ and $0 \%$, respectively. When the elder $+\widehat{\partial} \mathrm{PBH}$ chicken nucleus been reintroducing into breeding, it was observed in the $+/ \overbrace{}^{\text {th }}$ Kambro chicken nucleus there is fluctuation followed by an increase in upper and lower limits of accuracy to $95 \%$ and $75 \%$, respectively. It can be concluded that for the Broiler selection index, there is an increase in the lower limit of 
accuracy by $15 \%$ in the $5^{\text {th }}$ Kambro chicken nucleus against the nucleus of selected elders chicken, which indicates a decrease in the range of accuracy fluctuations by 15 points. According to this discovery, it is pretty practical to use an outbreeding technique in increasing the accuracy of genomic prediction of the three selection model scenarios was known. Implicitly, these three selection model scenarios reveal the fluctuation patterns uniformity from the accuracy result. The accuracy result $\left(r_{50}\right)$ over 50 generations shows that scenario_1 is the most suitable for selecting $\widehat{\delta} 5^{\text {th }}$ Kambro chicken and $\delta$ Pelung elders chickens using the Broiler selection index (Figure 2).

For the Pelung selection index, in the nucleus of the $\rightarrow$ BC500 and $\delta$ PBH selected elder chickens, three selection model scenarios have an accuracy limitation in upper and lower by $95 \%$ and $50 \%$, in order with an extreme fluctuation in each generation. In the $\$ / \delta 1^{\text {st }}$ and $2^{\text {nd }}$ Kambro chicken nucleus, the fluctuation is still happened, followed by the increase of accuracy's lower limit to $65 \%$ and $70 \%$, respectively. In the $9 / \hat{\sigma} 3^{\text {rd }}$ and $4^{\text {th }}$ Kambro chicken nucleus begin to achieve stability, followed by a decrease in the upper accuracy limit to $80 \%$. In addition, extreme lower accuracy limits of $-20 \%$ and $0 \%$ were detected, respectively. When the $q / \hat{O} \mathrm{PBH}$ elders chicken nucleus being reintroduced in the breeding, it is observed that $q / \hat{\sigma} 5^{\text {th }} \mathrm{Kambro}$ chicken nucleus fluctuations occur followed by an increase in the upper and lower limit of accuracy to $90 \%$ and $70 \%$, respectively.

To conclude, for the Pelung selection index, the upper limit of $5^{\text {th }}$ Kambro nucleus accuracy decreased by $5 \%$ against the nucleus of selected elders chicken meanwhile, there is a $20 \%$ increase for the lower limit. The fluctuation of the upper-and lower limit indicates a derivation in the range of accuracy by 25 points. According to this discovery, using an outbreeding technique to increase genomic prediction accuracy from the three selection model scenarios is pretty practical. In the entire perspective, these three selection model scenarios show a uniformity of fluctuation pattern from obtaining accuracy. According to the acquisition of accuracy $\left(r_{50}\right)$ of 50 generations, it is showing that scenario_1 is the most suitable for selecting $\delta^{\lambda} 5^{\text {th }}$ Kambro chicken and $\widehat{\delta}$ Pelung elders chickens using the Pelung selection index (Figure 2).

The decrease in the accuracy fluctuating range from the three selection model scenarios in the Pelung selection index is more significant than Broiler. However, the lower and upper limits of the $5^{\text {th }}$ Kambro chicken nucleus accuracy for the Pelung selection index are lessened than Broiler. On the other hand, in the lower limit of elders, chicken nucleus accuracy for Broiler index selection are higher than Pelung. The conclusion is that the use of the Broiler selection index is adequate than Pelung. The Pelung selection index assigns a higher value to the FEML (5) and TL (5) phenotype characters, while the Broiler selection index to FCR (5), BW49D (4), and BW56D (4). According to this discovery, weighting affects the accuracy of the Gama Ayam Kambro three selection model scenario. Through the contrast of upper and lower accuracy limit between the elders chicken nucleus and $5^{\text {th }}$ Kambro, also known as the use/utilization of outbreeding techniques, in general, are pretty effective in increasing the accuracy of genomic predictions of the three selection model scenarios for the two selection index. The selection index of the three selection model scenario shows the uniformity of fluctuation pattern from the accuracy result. According to the acquisition of accuracy ( $r 50)$ of 50 generations, it is showing that scenario_1 with genomic selection design is the most applicable for selecting $\widehat{\delta} 5^{\text {th }}$ Kambro and elder $\widehat{\delta}$ Pelung chickens, either using the Broiler or Pelung selection index, under the Gama Ayam Kambro breeding scheme. 

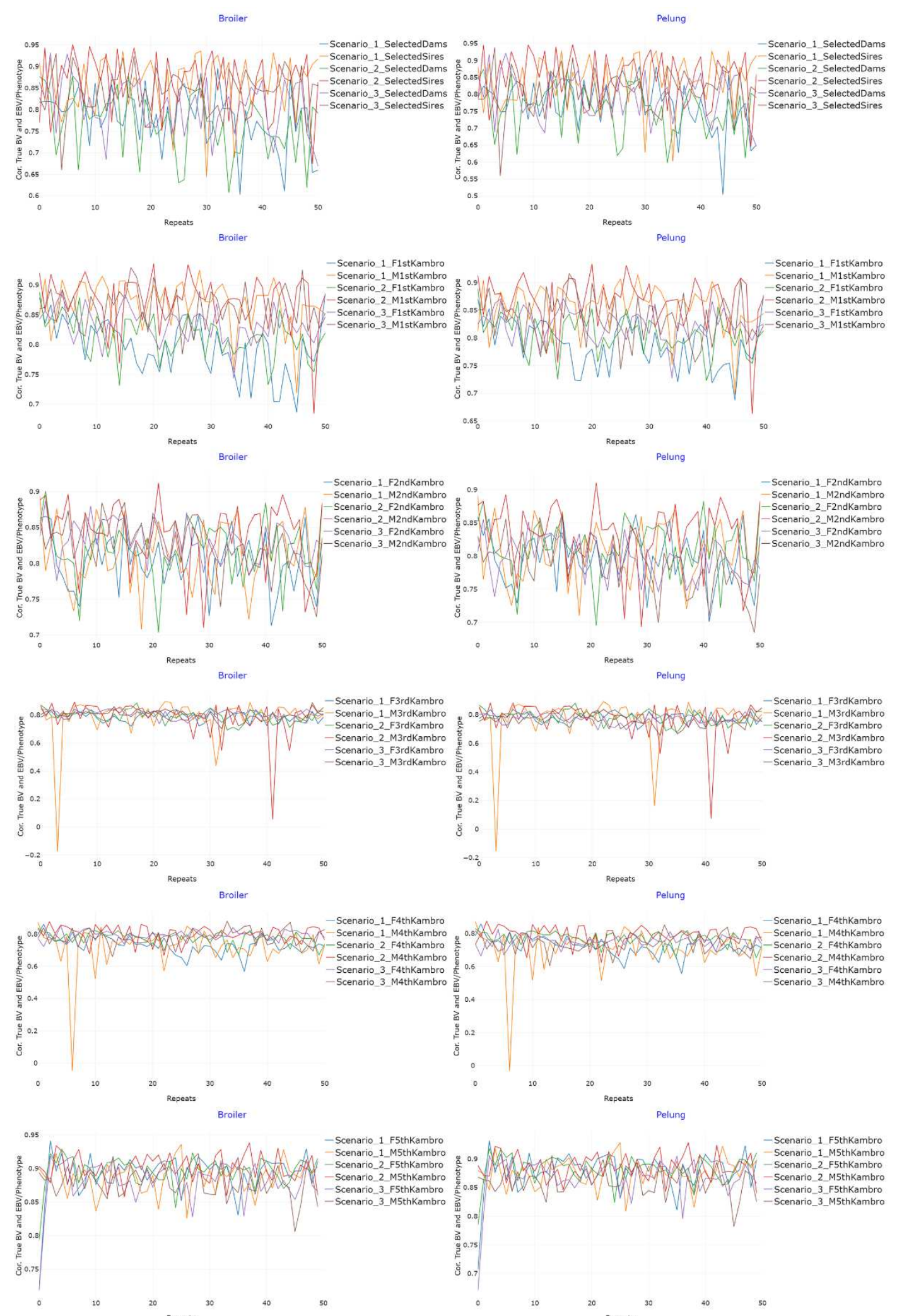

Fig 2. Accuracy of $r($ Skenario, TBV) from the Gama Ayam Kambro three selection model scenarios. MoBPSweb (2021)

An overview regarding the $F$ coefficient and weekly kinship of the three selection model scenarios in the Gama Ayam Kambro selective breeding program is described in each chicken nucleus, starting from the elders to the litters (Figure 3). For the $F$ coefficient, in the nucleus of selected $\odot$ BC500 and $\delta$ PBH elders, it is known that three selection scenarios have upper and lower limits of 0.3 and 0 , respectively, with a linear increase pattern per week. In the $+/ \widehat{\delta} 1^{\text {st }}$ Kambro nucleus, an increasing linear pattern is still detected, followed by a decrease in the upper limit of the $F$ coefficient to 0.25 . Meanwhile, the $+1 / \delta 2^{\text {nd }} \mathrm{Kambro}$, an increased linear pattern followed by an increase in the upper and lower limits to 0.42 and 0.26 , respectively. In the $+/ \delta 3^{\text {rd }}$ and $4^{\text {th }}$ Kambro nucleus, there is an $F$ coefficient of scenario 1 with scenario 2 and scenario_3. As a result, scenario_1 has a higher increasing pattern of the $F$ coefficient than scenario_2 and 
scenario 3. In the $3^{\text {rd }}$ Kambro nucleus, an increase in upper and lower limits of the $F$ coefficient was detected to 0.52 and 0.4 , respectively. Meanwhile, an increase in the $4^{\text {th }}$ Kambro nucleus was detected to 0.58 and 0.5 , respectively. As the $+/ \delta \mathrm{PBH}$ elders nucleus was reintroduced to breeding, it was observed that the $\$ / \delta^{\text {th }}$ Kambro nucleus fluctuated and followed by a decrease in the upper and lower limits of the $F$ coefficient to 0.035 and 0.005 , respectively (Figure 3 ).

According to upper and lower limits, there is a weekly increase in the $F$ coefficient from the $2^{\text {nd }}$ Kambro to the $4^{\text {th }}$ Kambro nucleus. In summary, there is a decrease in the upper limit of the $5^{\text {th }}$ Kambro nucleus $F$ coefficient by 0.265 points against the nucleus of the selected elders, while for the lower limit, there is an increase of 0.005 points. In addition, this also indicates a fluctuating range decrease in the $F$ coefficient by 0.04 points. According to these findings, using the outbreeding technique effectively reduces the $F$ coefficient of the three selection model scenarios. There is a separation of the $F$ coefficient of scenario_1 with scenario_2 and scenario_3 in the $3^{\text {rd }}$ and $4^{\text {th }}$ Kambro chicken nucleus. At least three factors most likely underlie this occurrence: the choice of broodstock, crossing techniques, and the selected design. It has been recognized that the $F$ coefficient of the $2^{\text {nd }}$ Kambro's nucleus has reached the relatively high relative limit to the nucleus of the elders or $1^{\text {st }}$ Kambro chicken. It is suspected to amplify the homozygotic allele fraction when the selected $2^{\text {nd }}$ Kambro is crossed with each other based on the common inbreeding technique to form + \& $/ 3^{\text {rd }}$ Kambro nucleus. A similar procedure was also applied in forming the nucleus of P/O $4^{\text {th }}$ Kambro. The selection model scenario factor is might play a passive role due to the accumulation of the two previous factors. According to $F$ coefficient $\left(F_{5000}\right)$ for 5000 weeks, it was found that scenario_ 1 with the most practical selection design for selecting $\delta 5^{\text {th }}$ Kambro and elder $\delta$ Pelung chicken under the Gama Ayam Kambro breeding scheme (Figure 3). 

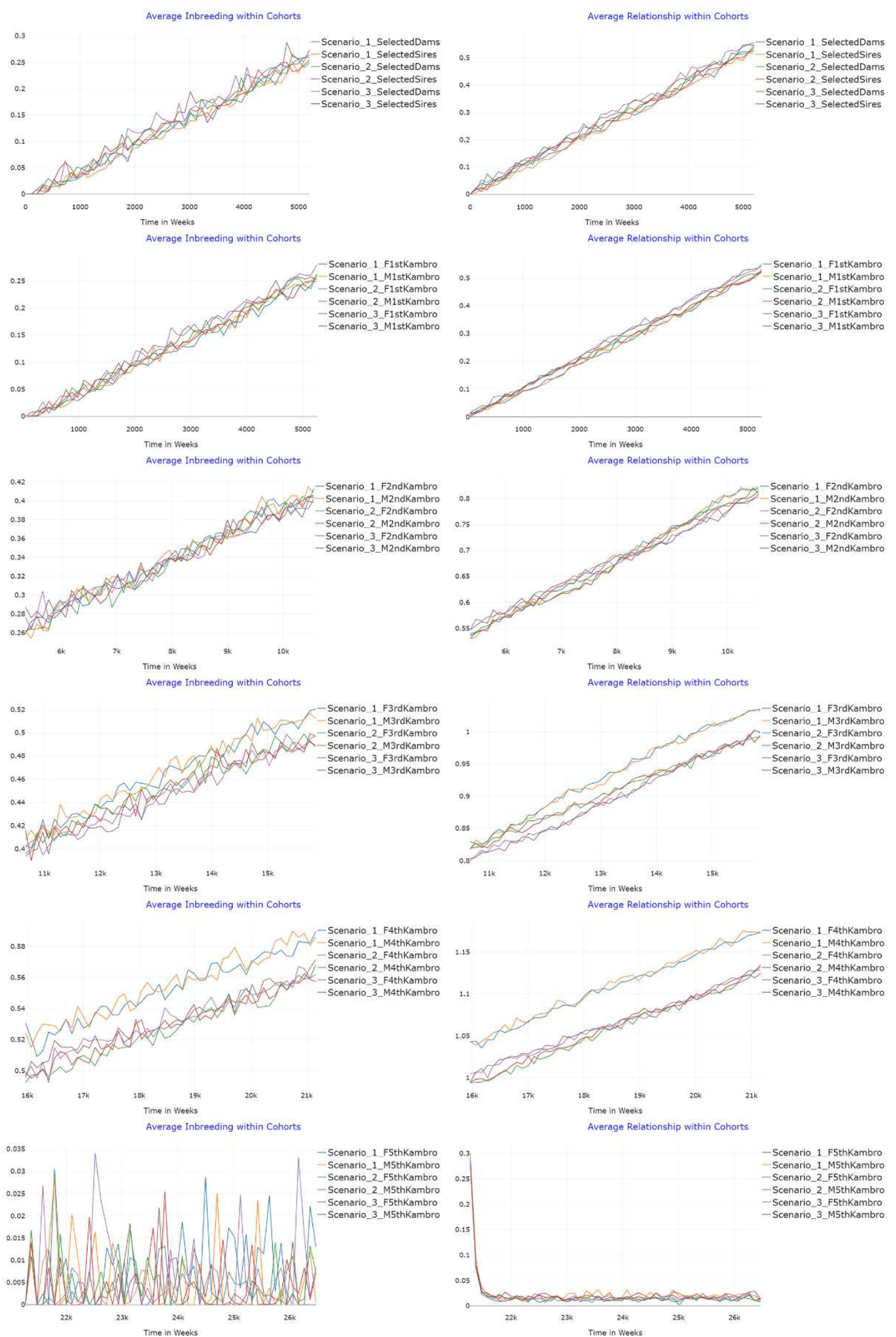

Fig 3. The $F$ coefficient and weekly kinship of the Gama Ayam Kambro three selection model scenario. MoBPSweb (2021).

In terms of kinship, in the nucleus of the selected 9 BC500 $\curvearrowright$ PBH and elders, it has known that the three selection model scenarios have upper and lower limits of 0.5 and 0 , respectively, with a linear increase pattern per week. In the $Q / \widehat{O} 1^{\text {st }}$ Kambro nucleus, the increasing linear pattern is still detected without changing the kinship's upper and lower limits. In $Q / \widehat{\delta} 2^{\text {nd }}$ Kambro nucleus, a linear pattern was improved, followed by an increase in the upper and lower to 0.8 and 0.55 , respectively. In the $q / \widehat{\gamma} 3^{\text {rd }}$ and $4^{\text {th }}$ Kambro nucleus, there is a separation of kinship between scenario_1 with scenario_2 and scenario_3. Scenario_1 has a higher increasing kinship pattern than scenario_2 and scenario_3. In the $3^{\text {rd }}$ Kambro nucleus, there is an uplift in the upper and lower limits of kinship to $\overline{1}$ and 0.8 , respectively. 
Meanwhile, an increase in the $4^{\text {th }}$ Kambro nucleus was detected to 1.15 and 1 , respectively. When the

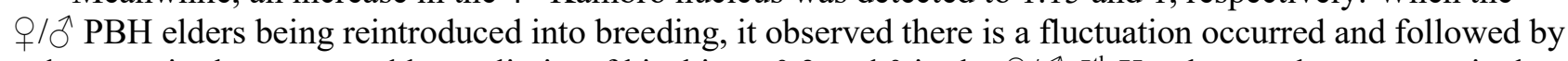
a decrease in the upper and lower limits of kinship to 0.3 and 0 in the $q / \delta 5^{\text {th }}$ Kambro nucleus, respectively (Figure 3). According to upper and lower limits, there is an increase in the weekly kinship starting from the $2^{\text {nd }}$ Kambro nucleus to the $4^{\text {th }}$ Kambro. Therefore, it can be assumed that there is a decrease in the $5^{\text {th }}$ Kambro kinship's upper limit by 0.2 points to the chosen elders chicken's nucleus. According to this discovery, using outbreeding techniques is quite effective in reducing the homozygotic allele fraction of the three selection model scenarios. The kinship strengthens the factors of broodstock and crossing technique as the $F$ coefficient separation of in the $3^{\text {rd }}$ and $4^{\text {th }}$ Kambro. In addition, it also proves the amplification of the homozygotic allele fraction, which leads to an increase in the $F$ coefficient. It is concluded that the cohort's close kinship increases the $F$ coefficient. According to the kinship $\left(R_{5000}\right)$ over 5000 weeks, it was found that scenario_1 with the genomic selection design is the most applicable for selecting $\sigma^{\lambda} 5^{\text {th }}$ Kambro and elders of $\widehat{\partial}$ Pelung chicken, under the Gama Ayam Kambro chicken breeding scheme (Figure 3).


Fig 4. The observed phenotype of AFE from the Gama Ayam Kambro three selection model scenarios. MoBPSweb (2021).

An overview of the observed phenotypes of AFE from the three selection model scenarios in the Gama Ayam Kambro selective breeding program with 50 generations was described per nucleus, from the elders to litters (Figure 4). In the nucleus of selected $q$ BC500 elders and $\stackrel{\wedge}{\mathrm{PBH}}$, the three selection model scenarios have upper and lower limits of AFE by 169 and 161 days, in order with a linear increase pattern per generation. The lowest AFE was detected in the same nucleus at scenario_2, while the highest is in

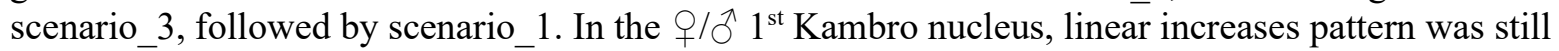
detected, followed by a decrease in the upper limit of AFE to 168 days. While in the $\$ / \widehat{\delta} 2^{\text {nd }} \mathrm{Kambro}$ nucleus, a linear increases pattern followed by an increase in the upper and lower limits to 171 and 166 days,

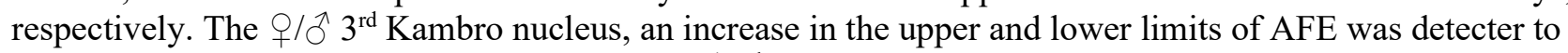
174 and 169 days, respectively. While in the $P / O 4^{\text {th }}$ Kambro nucleus, an increase was detected to 175 and 171 days, respectively. When the elders $\$ / \widehat{O} \mathrm{PBH}$ nucleus being reintroduced to breeding, it was observed in the $P / \widehat{\delta} 5^{\text {th }}$ Kambro nucleus there are a decrease in the upper and lower limits of AFE to 174 and 160days, respectively (Figure 4). According to upper and lower limits, there is an increase in AFE starting from the nucleus of $2^{\text {nd }}$ Kambro to $4^{\text {th }}$ Kambro. There is an increase in the upper limit of AFE $5^{\text {th }}$ Kambro nucleus by 5 points to the nucleus of the selected elders, while the lower limit decreased by 1 point. Moreover, that indicates an increase in AFE fluctuating range by 6 points. According to these findings, using the outbreeding technique reduces AFE from the three selection model scenarios. Based on $\operatorname{AFE}\left(A F E_{50}\right)$ 

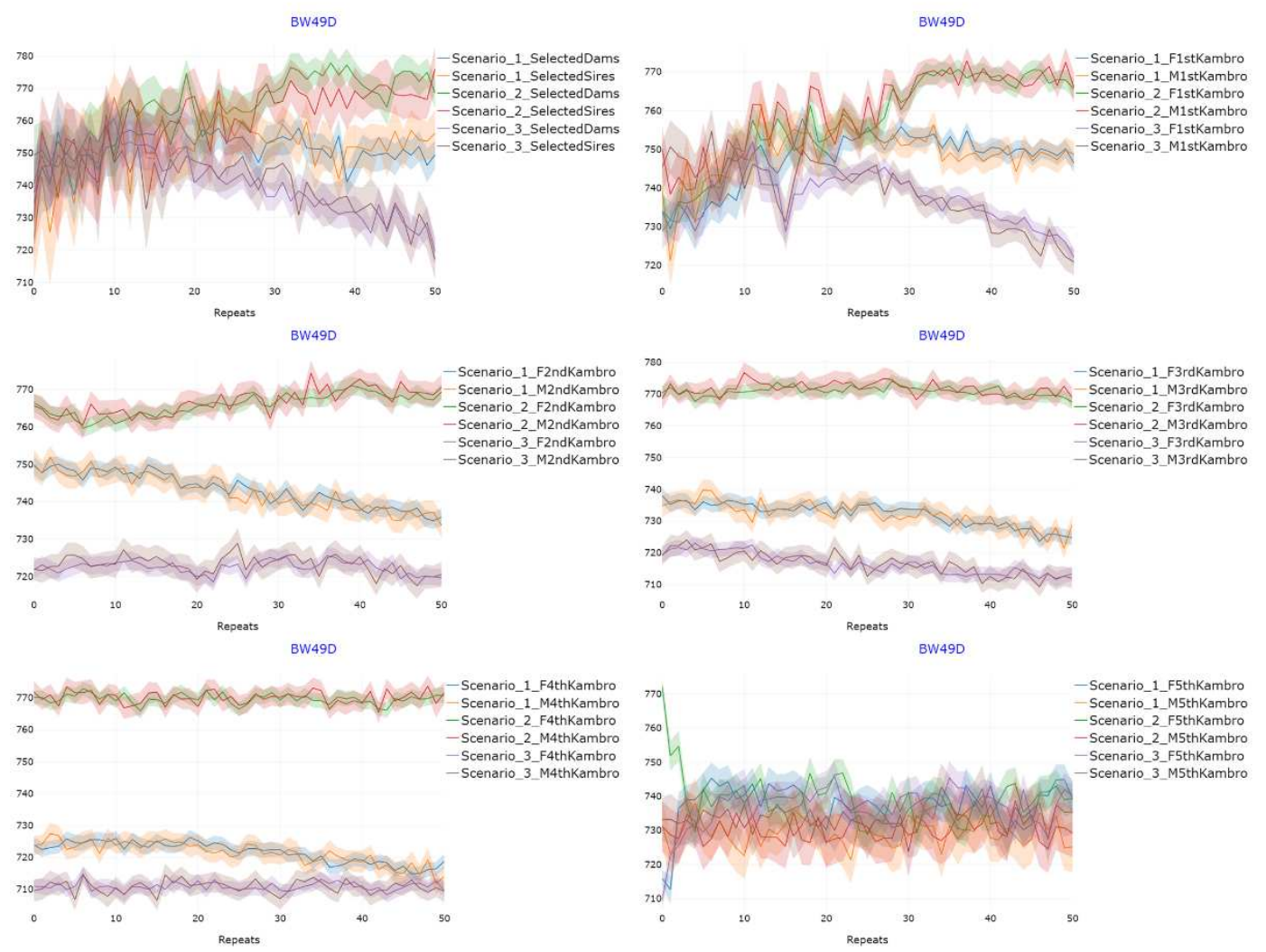

Fig 5. The observed phenotype of BW49D from the Gama Ayam Kambro three selection model scenarios. MoBPSweb (2021).

An overview of an observed phenotype of BW49D from the three selection model scenarios in the Gama Ayam Kambro selective breeding program with 50 generations was described per nucleus, starting from the elders to their litters (Figure 5). In the nucleus of selected elders 9 BC500 and $\curvearrowright$ PBH, it has known that the three selection model scenarios have upper and lower limits of BW49D of 780 and 710 grams, in order with fluctuations per generation. In the same chicken nucleus, the lowest BW49D was detected in scenario_3, while the highest was in scenario_2 and followed by scenario_1. The fluctuation in the nucleus of $q / \overline{\widehat{\sigma}} 1^{\text {st }}$ Kambro still happens, followed by a decrease in the upper limit and an increase in the BW49D's lower limit to 770 and 720 grams, respectively. Meanwhile, in the $+/ \delta 2^{\text {nd }}$ Kambro nucleus, an increase in the linear pattern was detected attenuation of fluctuations without changes in the upper and lower limits of BW49D relatively to the $1^{\text {st }}$ Kambro nucleus. Finally, in the $q / \delta 3^{\text {rd }}$ Kambro nucleus, it is indicated that there is an increase in the upper limit and a decreased lower limit of BW49D to 780 and 710 grams, respectively.

Meanwhile, in the $q / \delta 4^{\text {th }}$ Kambro nucleus, a decrease in the upper limit to 770 grams was detected. As the nucleus of $+/ \hat{\jmath} \mathrm{PBH}$ elders has been reintroducing into breeding, it has detected there was no change in the upper and lower limits of BW49D in the nucleus of $q / \delta 5^{\text {th }}$ Kambro. In the nucleus of $q / \lambda 5^{\text {th }}$ Kambro, fluctuations, and convergence of the upper and lower limits of BW49D were detected from the three selection model scenarios (Figure 5). According to upper and lower limits, there are no changes in the BW49D from the $1^{\text {st }}$ Kambro nucleus to the $4^{\text {th }}$ Kambro. It summarizes a decrease in the BW49D upper limit of the $5^{\text {th }}$ Kambro nucleus by 10 points to the nucleus selected elders, while there was no change for the lower limit. Otherwise, it indicates a decrease in the fluctuating range of BW49D by 10 points. In the $5^{\text {th }}$ Kambro nucleus, there was a convergence of upper and lower limits in the range of 750 and 710 grams, respectively. According to these findings, outbreeding techniques are less effective in increasing BW49D from the three selection model scenarios. Based on BW49D (BW49D50) over 50 generations, found that scenario_3 (PHEN) and scenario_2 (BV) are the most suitable for selecting $\sigma^{\lambda} 5^{\text {th }}$ Kambro chickens and $\delta$ Pelung elder chickens, respectively under the Gama Ayam Kambro breeding scheme (Figure 5). 

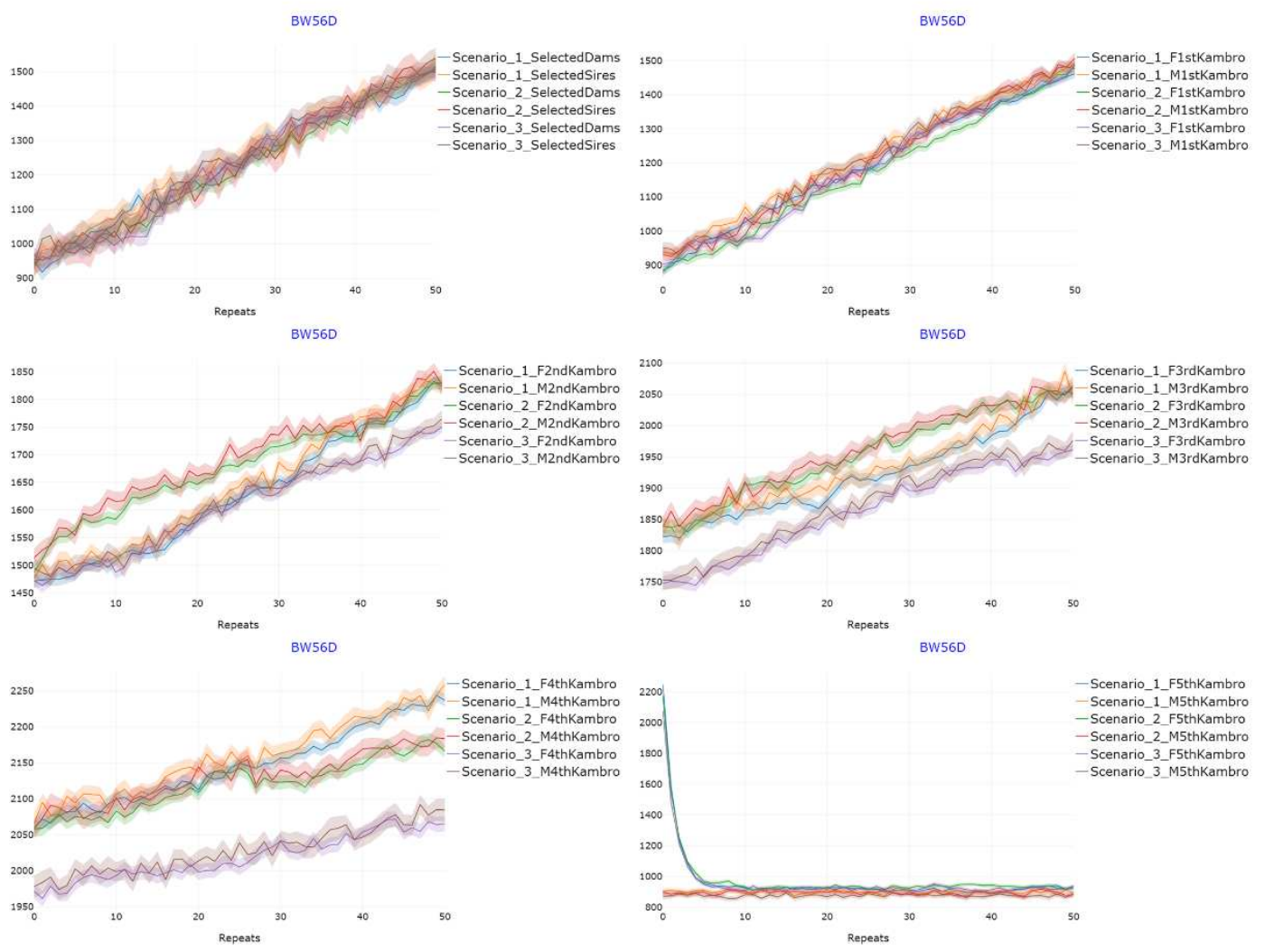

Fig 6. The observed phenotype of BW56D from the Gama Ayam Kambro three selection model scenarios. MoBPSweb (2021).

An overview of an observed phenotype of BW56D from the three selection model scenarios in the Gama Ayam Kambro selective breeding program with 50 generations was described per nucleus, from the elders to litters (Figure 6). In the nucleus of selected $\$$ BC500 and $\delta$ PBH elders, the three selection model scenarios have upper and lower limits of BW56D by 1500 and 900 grams, in order with increasing linear pattern. In the nucleus of $Q /{ }^{\Uparrow} 1^{\text {st }} \mathrm{Kambro}$, an increasing linear pattern is detected without changes in the upper and lower limits of BW56D. In the nucleus of $P /{ }^{\widehat{2}} 2^{\text {nd }} \mathrm{Kambro}$, an increasing linear pattern has been detected, followed by an increase in upper and lower limits of BW56D to 1850 and 1450 grams, respectively. In the nucleus of O $/ \widehat{\delta} 3^{\text {rd }}$ Kambro, a detected increase of upper and lower limits of BW56D to 2100 and 1750 grams, respectively. In the nucleus of $Q /{ }^{\circ} 4^{\text {th }} \mathrm{Kambro}$, an increase is detected in the upper and lower limits to 2250 and 1950 grams, respectively. The lowest of BW56D in the same nucleus is in scenario_3, whereas the highest is in scenario_1 and then scenario_2. When the nucleus of $9 / \widehat{P B H}$ elders reintroduced into the breeding, it was observed that there is a decrease in upper and lower limits of BW56D to 2200 and 900 grams in the $q / \delta^{t} 5^{\text {th }}$ Kambro nucleus, respectively (Figure 6). Based on upper and lower limits, there is an increase of BW56D starting from the nucleus of $2^{\text {nd }}$ Kambro to $4^{\text {th }}$ Kambro. The conclusion is an increase in the upper limit of BW56D in the $5^{\text {th }}$ Kambro nucleus by 700 points towards the elder's nucleus, whereas, for the lower limit, there was a decrease of 100 points. Moreover, that indicates there is a decrease fluctuating range by 200 points. According to these findings, the outbreeding technique effectively increases BW56D from the three selection model scenarios. Based on BW56D $\left(B W 56 D_{50}\right)$ over 50 generations, scenario_1 (GEN) and scenario_3) are the most applicable for selecting $\delta^{\lambda} 5^{\text {th }}$ Kambro chickens and $\widehat{\partial}$ Pelung chickens, respectively under the Gama Ayam Kambro breeding scheme (Figure 6). 
EN

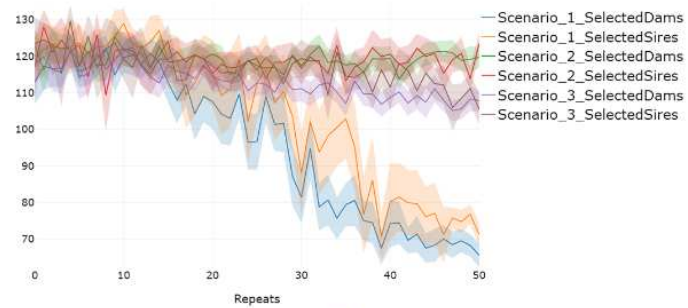

EN

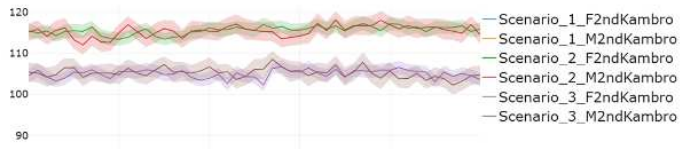

90

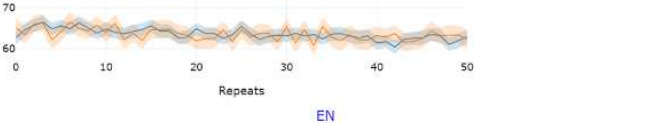

EN

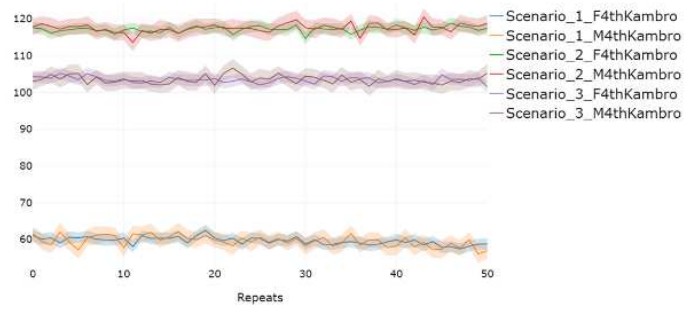

EN
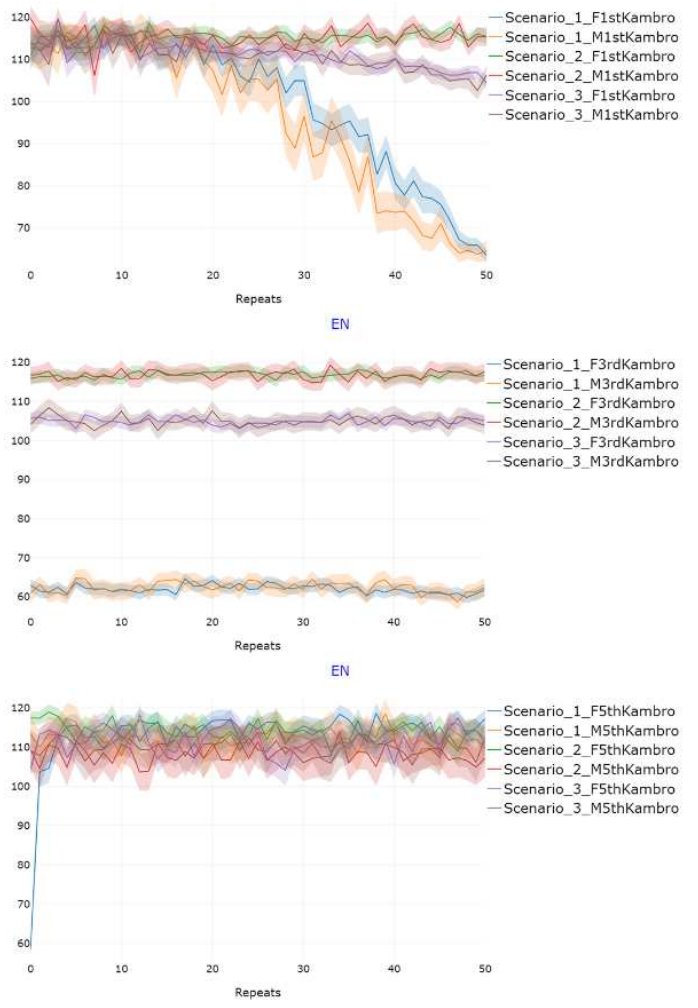

Fig 7. The observed phenotype of EN from the Gama Ayam Kambro three selection model scenarios. MoBPSweb (2021).

An overview of an observed phenotype of EN from the three selection model scenarios of the Gama Ayam Kambro selective breeding program with 50 generations was described per nucleus, from the elders to their litters (Figure 7). In the nucleus of selected $\odot$ BC500 and $\widehat{\partial}$ PBH elders, the three selection model scenarios have an upper and lower limit of EN by 130 and 70 items/day, with fluctuations in each generation. In the same nucleus, the lowest EN was detected in scenario_1, while the highest is in scenario_2, followed

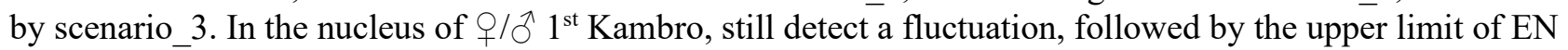
decreasing to 120 items/day. Meanwhile, in the nucleus of $P /{ }^{\lambda} 2^{\text {nd }} \mathrm{Kambro}$, an increasing linear pattern with

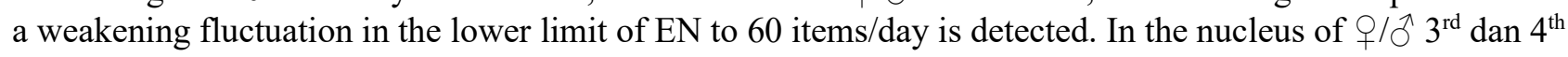
Kambro, an increasing linear pattern without any change on upper and lower limits of EN relatively to the

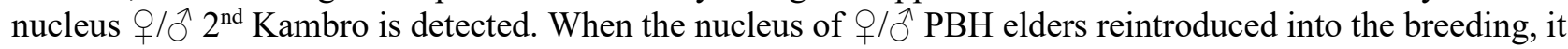
observed that in the nucleus of $O / \delta 5^{\text {th }}$ Kambro, there is any change in upper and lower limits of EN (Figure 7). Based on upper and lower limits, there is no change in EN from the nucleus of $1^{\text {st }}$ Kambro to $4^{\text {th }}$ Kambro. It summarizes a decreasing upper and lowers limits of the EN nucleus of $5^{\text {th }}$ Kambro by 10 points towards the nucleus of selected elders. In addition, it also indicates a stable range of EN fluctuations. According to these findings, using the outbreeding technique is less effective in increasing EN from the three selection scenarios. Based on EN $\left(E N_{50}\right)$ over 50 generations, scenario_3 (PHEN) and scenario_2 (BV) are suitable for selecting $\delta^{\lambda} 5^{\text {th }}$ Kambro chickens and $\delta^{\lambda}$ Pelung elder chickens, respectively under the Gama Ayam Kambro breeding scheme (Figure 7). 


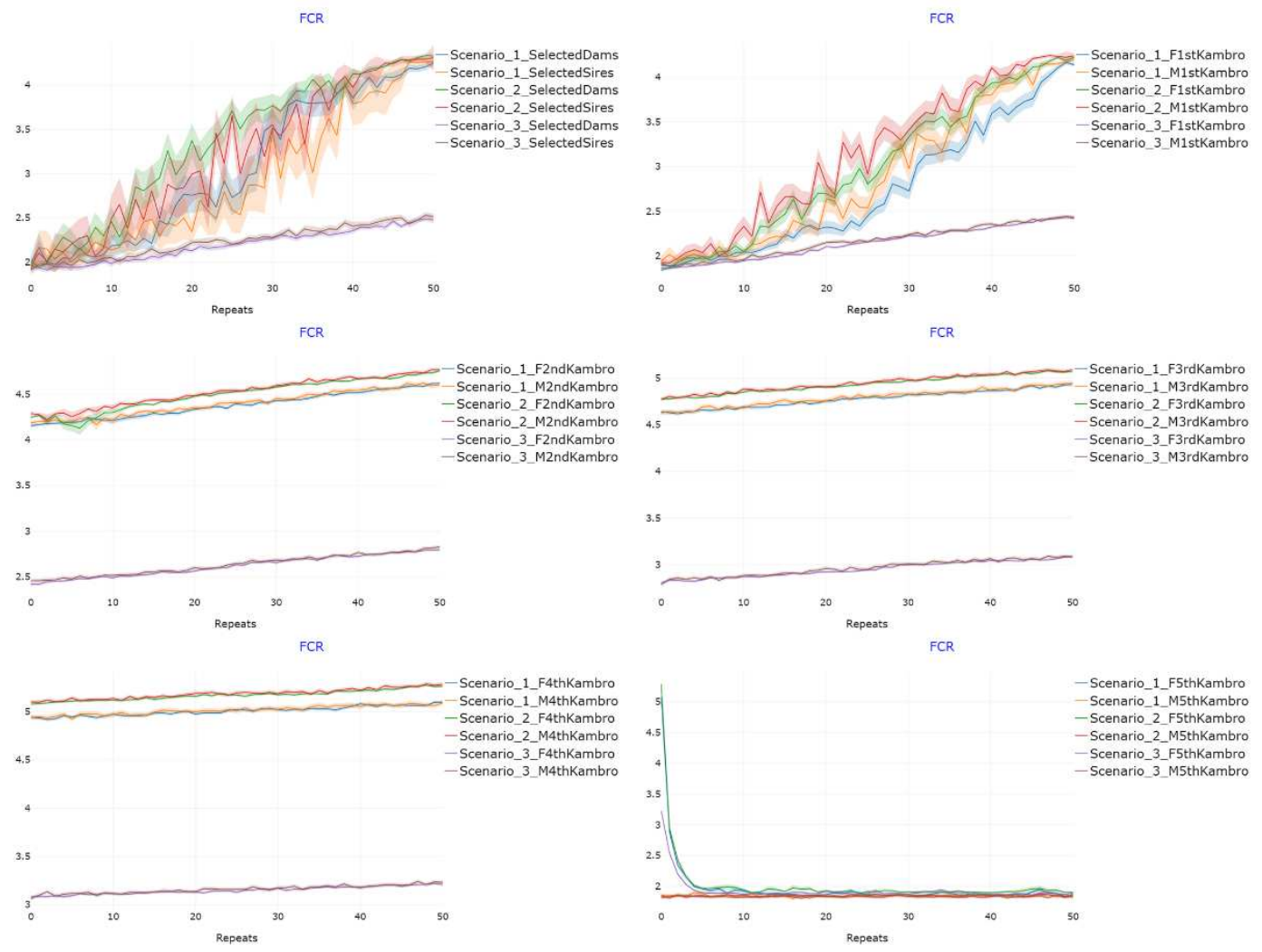

Fig 8. The observed phenotype of FCR from the Gama Ayam Kambro three selection model scenarios. MoBPSweb (2021).

An overview of an observed phenotype of FCR from the three selection model scenarios of the Gama Ayam Kambro selective breeding program with 50 generations was described per nucleus, from the elders to their litter (Figure 8). In the nucleus of selected $q$ BC500 and $\widehat{\partial}$ PBH elders, the three selection model scenarios have upper and lower limits of FCR by 4 and $2 \mathrm{~kg} / \mathrm{kg}$, in order with fluctuations in each generation. In the same nucleus, the lowest FCR was detected in scenario_3, while the highest is in scenario_2, followed by scenario_1. The nucleus of $q / \widehat{O} 1^{\text {st }}$ Kambro has still detected fluctuation without any changes in the upper and lower limits of FCR. Meanwhile, in the nucleus of $\$ / \delta 2^{\text {nd }} K a m b r o$, an increasing linear pattern with a weakening fluctuation followed by an increase in the upper limit of FCR to 4,5 and $2,5 \mathrm{~kg} / \mathrm{kg}$, respectively. In the nucleus of $P / \delta^{\text {rd }}$ dan $4^{\text {th }} \mathrm{Kambro}$, an increasing linear pattern is detected, followed by an increase of upper and lower limits of FCR to 5 and $3 \mathrm{~kg} / \mathrm{kg}$, respectively. When reintroduced the nucleus of $q / \widehat{P B H}$ elders into the breeding, it observed that in the nucleus of $+/ \delta 5^{\text {th }}$ Kambro, there is a decrease in the lower limit of FCR to $2 \mathrm{~kg} / \mathrm{kg}$ (Figure 8). Based on upper and lower limits, there is an increase in FCR that started from the nucleus of $2^{\text {nd }}$ Kambro to $4^{\text {th }}$ Kambro. It can be concluded that there is an increase in the FCR upper limit in the nucleus of $5^{\text {th }}$ Kambro by 1 point towards the nucleus of the elders, while there is no change for the lower limit. In addition, it also indicates an increased fluctuating range of FCR by 1 point. According to these findings, using the outbreeding technique is less effective in lowering the FCR of the three selection model scenarios. Based on FCR $\left(F C R_{50}\right)$ over 50 generations, scenario_1 (GEN) and scenario_3 (PHEN) are the most suitable for selecting $\widehat{\partial} 5^{\text {th }}$ Kambro chickens and $\widehat{\partial}$ Pelung elder chickens, respectively under the Gama Ayam Kambro breeding scheme (Figure 8). 

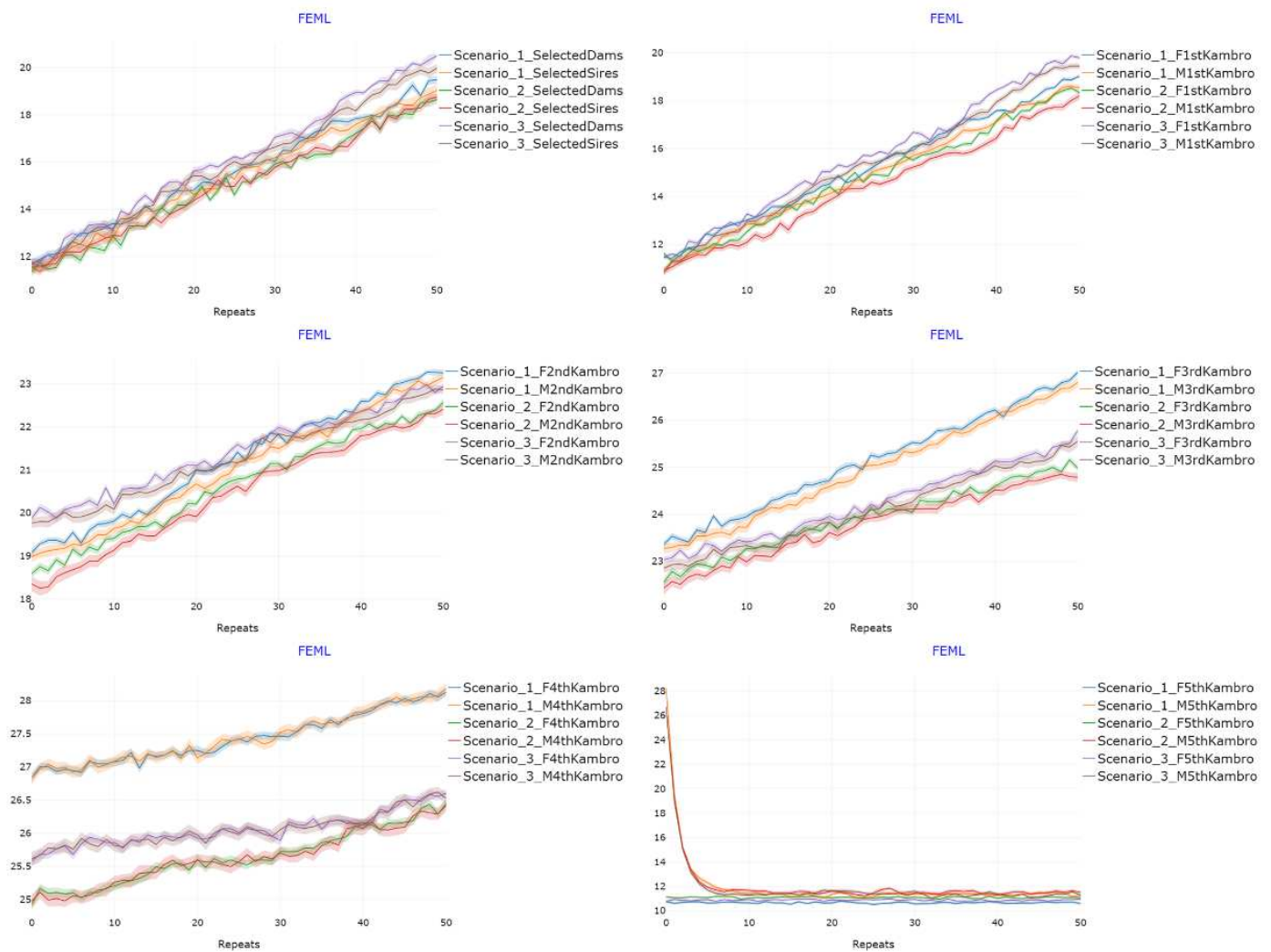

Fig 9. The observed phenotype of FEML from the Gama Ayam Kambro three selection model scenarios. MoBPSweb (2021).

An overview of an observed phenotype of FEML from the three selection model scenarios of the Gama Ayam Kambro selective breeding program with 50 generations was described per nucleus, from the elders to their litters (Figure 9). In the nucleus of selected $q$ BC500 and $\widehat{\partial}$ PBH elders, the three selection model scenarios have upper and lower limits of FEML by 20 and $12 \mathrm{~cm}$, respectively, with the increasing linear in each generation. The lowest FEML is detected in the same nucleus at scenario_2, while the highest is at

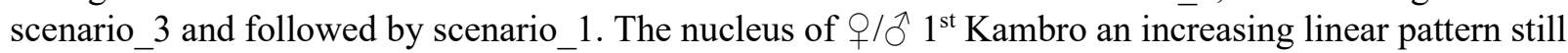
detected without any changes in the upper and lower limits of FEML. Meanwhile, in the nucleus of $q / \widehat{\gamma} 2^{\text {nd }}$ Kambro, an increasing linear pattern followed by increasing the upper and lower limits of FEML to 23 and


followed by increasing the upper and lower limits of FEML to 27 and $23 \mathrm{~cm}$, respectively. Meanwhile, in the nucleus of $+/ \delta^{\text {th }} 4^{\text {th }}$ Kambro, followed by increasing upper and lower limits of FEML by 28 and $25 \mathrm{~cm}$, respectively. When the nucleus of $\$ / \delta$ PBH elders being reintroduced into the breeding, it observed a decrease in the lower limits of FEML to $10 \mathrm{~cm}$ in the nucleus of $+/ \delta^{\text {th }}$ Kambro (Figure 9). Based on upper and lower limits, an increasing FEML started from the nucleus $2^{\text {nd }}$ Kambro to $4^{\text {th }}$ Kambro. It summarizes an increase in the upper limit of FEML in the nucleus of $5^{\text {th }}$ Kambro to 8 points towards the nucleus of elders, which is a decrease by 2 points for the lower limit. In addition, it indicates an increase in the fluctuating range of FEML by 10 points. Based on these findings, the outbreeding technique effectively increases the FEML from the three selection model scenario. Based on FEML (FEML50) over 50 generations, scenario_2 (BV) and scenario_3 (PHEN) are the most suitable for selecting $\delta 5^{\text {th }}$ Kambro chickens and $\delta$ Pelung elder chickens, respectively under the Gama Ayam Kambro breeding scheme (Figure 9). 

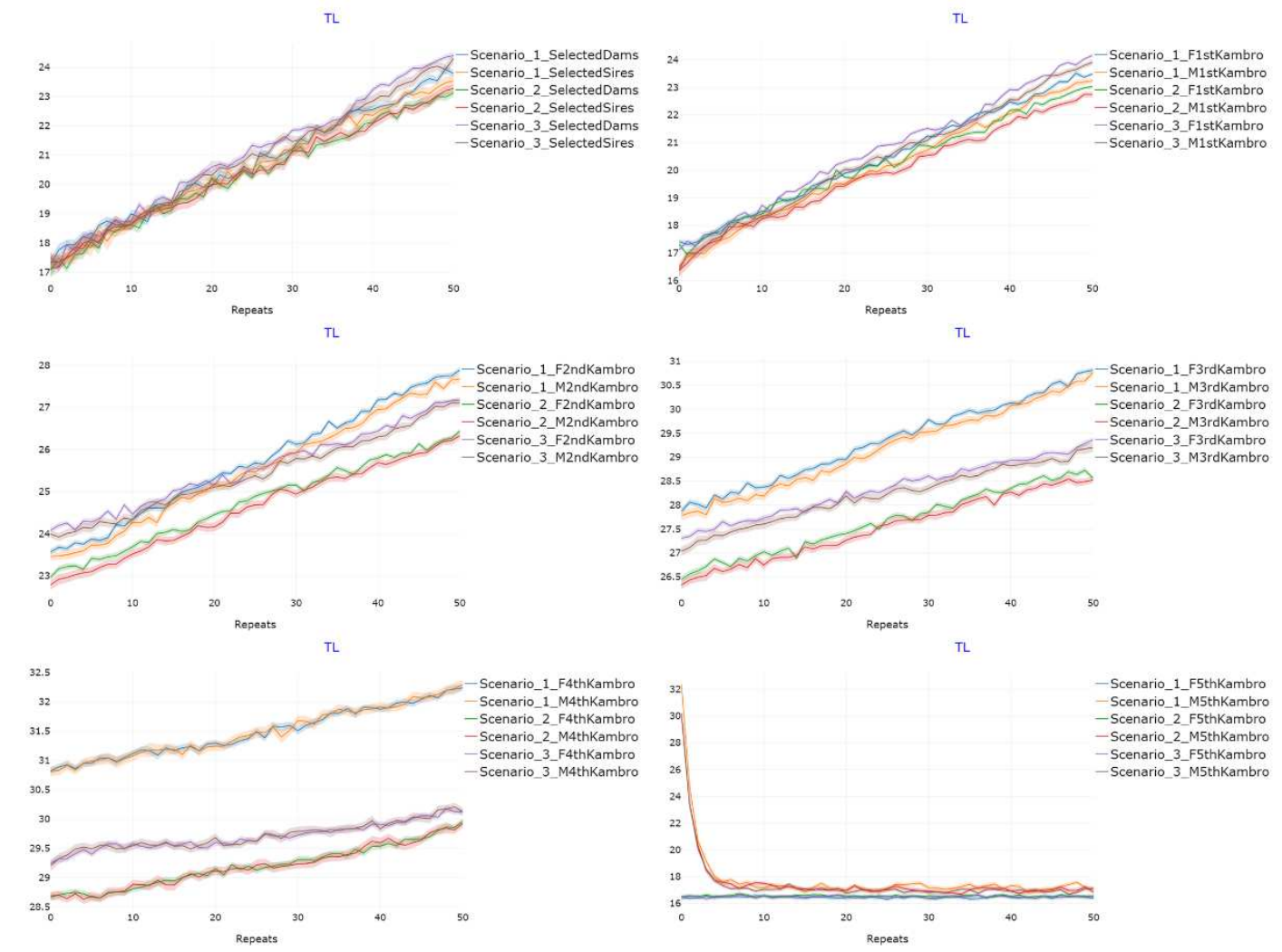

Fig 10. The observed phenotype of TL from the Gama Ayam Kambro three selection model scenarios. MoBPSweb (2021).

An overview about an observed phenotype of TL from the three selection breeding scenarios of the Gama Ayam selective breeding program with 50 generations was described per nucleus, starting from the elders to their litters (Figure 10). In the nucleus of selected $q$ BC500 and $\widehat{\jmath}$ PBH elders, the three selection model scenarios have upper and lower limits of TL by 24 and $17 \mathrm{~cm}$, in order with an increasing linear pattern in each generation. It is detected that the lowest TL is in the same nucleus at scenario_2, the highest is

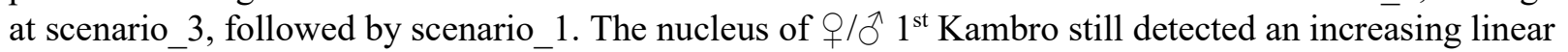
pattern, followed by a decrease in the lower TL limit by $16 \mathrm{~cm}$. Meanwhile, in the nucleus of $O / \delta^{1} 2^{\text {nd }}$ Kambro, an increasing linear pattern is detected, followed by an increase of TL's upper and lower limits to 28

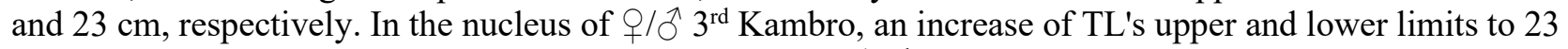
and $26.5 \mathrm{~cm}$ is detected, respectively. In the nucleus of $+/{ }^{1} 4^{\text {th }}$ Kambro, TL's upper and lower limits

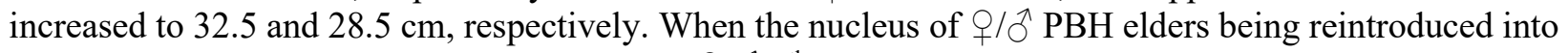
the breeding, it was observed in the nucleus of $Q / \delta 5^{\text {th }}$ Kambro there is a decrease in upper and lower limits of TL to 32 and $16 \mathrm{~cm}$, respectively (Figure 10). Based on upper and lower limits, there is an increase in TL starting from the nucleus of $2^{\text {nd }}$ Kambro to $4^{\text {th }}$ Kambro. It summarizes an increasing upper limit of TL in the nucleus of $5^{\text {th }}$ Kambro by 8 points towards the nucleus of the elders, while there is a decrease to 1 point for the lower limit.

Meanwhile, it indicates an increased fluctuating range of TL by 9 points. According to these findings, the outbreeding technique effectively increases TL from the three selection model scenarios. Therefore, based on TL $\left(T L_{50}\right)$ over 50 generations, scenario_3 with phenotype design selection is most suitable for selecting the

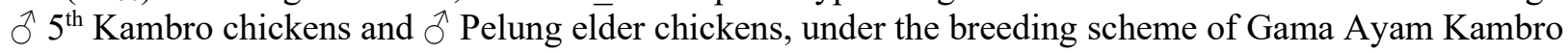
(Figure 10).

The economic parameters are designed based on the field operational cost in the Gama Ayam Kambro selective breeding program with an interest rate of $2.5 \%$ and a EUR/Rp exchange rate of Rp 16.897 per 27 August 2021 (see Table 32, Appendix 16). The economic parameters were tested against the three selection model scenarios and analyzed internally by the MoBPSweb simulator. The economic parameters are projected for 50 breeding generations with operational cages in 100 units, and each will implement intensive maintenance management. For ten years, the projection economic parameters produced a total of 113.975 chickens which accumulated from each nucleus, from the elders to their litters. The total required operational cost is around \pm 4.79 billion rupiahs. The cost of operation is accumulated from three components which are genotyping, phenotyping, and housing. The total genotyping cost required is \pm 1.89 billion rupiahs, while the 
phenotyping and housing cost are around \pm 1.89 and \pm 0.995 billion rupiah, respectively (see Supp.File_SIM08). Counting the inflation rate and interest rate of rupiah each year requires an operational cost of around \pm 500 million rupiahs for applying the breeding scheme and selection procedure of Gama Ayam Kambro. These findings summarized that the crossing technique, selection model scenario, and breeding scheme affect the achievements of the Gama Ayam Kambro selective breeding program. From the aspects of accuracy, it concluded that the selection of Pelung and $5^{\text {th }}$ Kambro males would be more optimal when using scenario_1 with the genomic selection design for both Broiler and Pelung selection index. The same conclusion also applies to the $F$ coefficient and kinship. Generally, the outbreeding technique is quite effective in increasing the accusation and controlling the $F$ coefficient.

However, different things concluded regarding the phenotype characters of AFE, BW49D, EN, and FCR. Meanwhile, for the phenotype characters of FEML, TL, and BW56D, the conclusion is that the outbreeding technique is quite effective for increasing those three achievements. Different results were obtained for each phenotype character related to the selection model scenario. The phenotype characters of AFE and FEML concluded that the selection of $5^{\text {th }}$ Kambro and Pelung males would be more optimal when using scenario_2 (BV) and scenario_3 (PHEN), respectively. Meanwhile, for the BW49D and EN phenotype characters, precocity applies. Different things concluded regarding the selection of $5^{\text {th }}$ Kambro and Pelung males for the phenotype characters of FCR and BW56D, which will be more optimal if using the scenario_1 (GEN) and scenario_3 (PHEN), respectively. However, the male selection will be more optimal for the $\bar{T} L$ phenotype character if using scenario_3 with phenotype design selection.

\section{CONCLUSIONS}

As well as accuracy, $F$ coefficient, kinship, and observed phenotype are determined by crossing technique, selection model scenario, and breeding scheme. The selection rate of $5^{\text {th }}$ Kambro and Pelung males can be optimized using the scenario_1 genomic selection design and outbreeding technique, both for Broiler and Pelung selection index. The selection model scenario and similar crossing technique are also quite effective in controlling the $F$ coefficient. However, the outbreeding technique is less effective for increasing the achievements of AFE, BW49D, EN, and FCR phenotype characters, otherwise for the phenotype characters of FEML, TL, and BW56D. For the phenotype characters of AFE and FEML, the selection of $5^{\text {th }}$ Kambro and Pelung males can be optimized by using scenario_2 (BV) and scenario_3 (PHEN), respectively.

Meanwhile, for the phenotype characters of BW49D and EN, reciprocal applies. The selection of $5^{\text {th }}$ Kambro and Pelung males for the phenotype characters of FCR and BW56D will be more optimal if using the scenario_1 (GEN) and scenario_3 (PHEN), respectively. Meanwhile, the phenotype characters of TL will be more optimal if scenario_3 with phenotype design selection is used for both. The projections of economics parameters show that the total operating costs required per year are around \pm 500 million rupiahs for the three selection model scenarios using Gama Ayam Kambro breeding scheme with 100 units of intensive maintenance management cages 50 breeding generations. Naturally, the projected operational costs must count the inflation rates and interest rates in rupiah per year. Technically, computation has many shortcomings; the number repetition, projections of economic parameters, outcome visualization, and analysis options are still limited. Aside from that, several selection procedures need to be improved, especially for marker density and particular kinship matrix for the ss(G)BLUP procedure. Nonetheless, the integration of MoBPSweb provides highly unique and unprecedented opportunities to forecast for the first time the possible outcome of a selective breeding program. In itself, this would help to breach further and handle both the complexity and limitations of the breeding program without necessarily having to comprise the gains, ethical risk, and operation costs.

Acknowledgments: Authors acknowledge the Gama Ayam Research Team for ensuring the completion of this project. Pusat Inovasi Agroteknologi and Faculty of Biology Universitas Gadjah Mada Daerah Istimewa Yogyakarta, Indonesia for facilitating the research. Pook, T. as a MoBPS conceptor and developer, and Animal Breeding and Genetics Group, Center for Integrated Breeding Research for allowing us to privately using the simulator in web version.

Authors' contributions: Mahardhika, I.W.S. and Hida, F.N.L. contribute by performing the research, analyzing the results, and preparing the manuscript for publication. Retnoaji, B., Widiyanto, S., Pook, T., and Daryono, B.S. contribute by conceiving the idea, supervising, and advising throughout the research. Pook, T., and Animal Breeding and Genetics Group, Center for Integrated Breeding Research contribute by cencepting and constructing the simulator.

Funding: The author received no specific funding for this work. 
Competing interests: The author has declared no competing interests exist.

Availability of data and materials: The selective parameters used in this study are included in this article [Supplemental File 1].

\section{REFERENCES}

Eriksson J, Larson G, Gunnarsson U, Bed'hom B, TIxier-Boichard M, Strömstedt L, Wright D, Jungerius A, Vereijken A, Randi E, Jensen P, Andersson L. 2008. Identification of the yellow skin gene reveals a hybrid origin of the domestic chicken. PLoS Genet 4(2): e1000010. https://doi.org/10.1371/journal.pgen.1000010

Daryono B S, Mushlih M, Perdamaian A B I. 2021. Crowing sound and inbreeding coefficient analysis of Pelung chicken (Gallus gallus domesticus). BIODIVERSITAS. 22(5): 2451-2457. https://doi.org/10.13057/biodiv/d220501

Daryono B S, Roosdianto I, Saragih, H T S S G. 2010. Pewarisan karakter fenotipe ayam hasil persilangan ayam Pelung dengan ayam Cemani. Jurnal Veteriner. 11(4): 257-263. https://ojs.unud.ac.id/index.php/jvet/article/view/3460

De Koning D J, Haley C S, Windsor D, Hocking P M, Griffin H, Morris A, Vincent J, Burt D W. 2004. Segregation of QTL for production traits in commercial meat-type chickens. Genet Res Camb. 83: 211220. https://doi.org/10.1017/S0016672304006846

Ferlito C, Respatiadi H. 2018. Reformasi kebijakan pada industri unggas di Indonesia. Center for Indonesia Policy Studies. https://doi.org/10.35497/271879

Goto T, Ishikawa A, Onitsuka S, Goto N, Fujikawa Y, Umino T, Nishibori M, Tsudzuki M. 2011. Mapping quantitative trait loci for egg production traits in an $\mathrm{F}_{2}$ intercross of Oh-Shamo and White Leghorn chickens. Animal Genetics. 42(6): 634-641. https://doi.org/10.1111/j.1365-2052.2011.02190.x

Hansen C, Yi N, Zhang Y M, Xu S, Gavora J, Cheng H H. 2005. Identification of QTL for production traits in chickens. Animal Biotechnology. 16(1): 67-79. https://doi.org/10.1081/ABIO-200055016

Henuk Y L, Bakti D. 2018. Benefits of promoting native chickens for sustainable rural poultry development in Indonesia. TALENTA Conference Series: Agricultural \& Natural Resources (ANR). 01: 069-076. https://doi.org/10.32734/anr.v1i1.98

Hodson R. 2017. Nature Outlook: Food security. Nature. 544(7651): Suppl., 27-04 (2017). http://www.nature.com/nature/outlook/food-security

Jennen D G J, Vereijken A L J, Bovenhuis H, Crooijmans R M P A, van der Poel J J, Groenen M A M. 2005. Confirmation of quantitative trait loci affecting fatness in chickens. Genet Sel Evol. 37: 215-228. https://doi.org/10.1051/gse:2004045

Kurnia R R, Lesmana I, Ernanto A R, Perdamaian A B I, Trijoko, Daryono B S. 2021. The association of follicle stimulating hormone receptor (FSHR) gene polymorphism of on egg productivity in hybrid chicken (Gallus gallus gallus, Linnaeus 1758). BIODIVERSITAS. 22(3): 1221-1226. https://doi.org/10.13057/biodiv/d220318

Li D Y, Zhang L, Smith D G, Xu H L, Liu Y P, et al. 2013. Genetic effects of melatonin receptor genes on chicken reproductive traits. Czech J Anim Sci. 58(2): 58-64. https://doi.org/10.17221/6615-CJAS

Mahardhika I W S, Habibah I, Perdamaian A B I, Trijoko, Daryono B S. 2021. Origin, phenotypes, and plumage coloration of golden Pelung chicken progenies (G. gallus, Linn.1758). Research Square. https://doi.org/10.21203/rs.3.rs-411024/v1

Mahardhika I W S, Chasnaurosyiqoh A R, Chohansandhika J, Sholiha F P, Indriarto N B, Daryono B S. 2020. Estimation of several commercial, phenotypic, and reproductive traits' performance using the quantitative genetic method for Kamper chicken line. Biogenesis. 8(2): 172-184. https://doi.org/10.24252/bio.v8i2.15648

Mahardhika I W S, Daryono B S, Dewi A A C, Hidayat S N, Firmansyah G I., et al. 2020. Phenotypic traits, egg productivity, and body weight performance of Gama Ayam BCl Kamper. Jurnal Peternakan. 17(1): 6-16. https://dx.doi.org/10.24014/jupet.v17i1:7331 
Mahardhika I W S, Daryono B S. 2019. Phenotypic performance of Kambro crossbreeds of female Broiler Cobb 500 and male Pelung Blirik Hitam. Buletin Veteriner Udayana. 11(2): 188-202. https://doi.org/10.24843/bulvet.2019.v11.i02.p12

Nataamijaya A G. 2010. Pengembangan potensi ayam lokal untuk menunjang peningkatan kesejahteraan petani. Jurnal Litbang Pertanian. 29(4): 131-138.

Nie Q-H, Fang M-X, Xie L, Shen X, Liu J, et al. 2010. Associations of ATGL gene polymorphisms with chicken growth and fat traits. J Appl Genet. 51(2): 185-191. https://doi.org/10.1007/BF03195726

Nie Q, Sun B, Zhang D, Luo C, Ishag N A, et al. 2005. High diversity of the chicken growth hormone gene and effects on growth and carcass traits. Journal of Heredity. 96(6): 698-703. https://doi.org/10.1093/jhered/esi114

Perdamaian A B I, Saragih H T S S G, Daryono B S. 2017. Effect of varying level of crude protein and energy on insulin-like growth factor-1 expression level in Indonesian hybrid chicken. Int J Poult Sci. 16(1): 1-5. https://doi.org/10.3923/ijps.2017.1.5

Perdamaian A B I, Trijoko, Daryono B S. 2017. Pertumbuhan dan keseragaman warna bulu ayam persilangan balik (BC2) hasil seleksi genetik persilangan ayam Pelung dengan ayam Pedaging. Jurnal Veteriner. 18(4): 557-564. https://doi.org/10.19087/jveteriner.2017.18.4.557

Pook T, Büttgen L, Ganesan A, Ha N-T, Simianer H. 2021. MoBPSweb: A web-based framework to simulate and compare breeding programs. Genes Genomes Genetics. 1(2): jkab023. https://doi.org/10.1093/g3journal/jkab023

Pook T, Schlather M, Simianer, H. 2020. MoBPS-modular breeding program simulator. Genes Genomes Genetics. 10(6): 1915-1918. https://doi.org/10.1534/g3.120.401193

Retnoaji B, Wulandari R, Nurhidayat L, Daryono B S. 2016. Osteogenesis study of hybrids of Indonesia's native chicken Pelung (Gallus gallus domesticus) with Broiler (Gallus gallus domesticus). Asian Journal of Animal and Veterinary Advances. 11: 498-504. https://doi.org/10.3923/ajava.2016.498.504

Saragih H T S G, Perdamaian A B I, Sadiman, Roosdianto I, Daryono B S. 2021. Plumage colours Stability in Inbreed Pelung Chicken. BIO Web of Conferences. 33: 01005. https://doi.org/10.1051/bioconf/20213301005

Saragih H T S, Viniwidihastuti F, Lembayu R P, Kinanthi A R, Kurnianto H, Lesmana I. 2019. Karakteristik fenotip ayam broiler eksotik, kampung, layer eksotik jantan, KUB-1 dan Pelung. JITV. 24(1): 9-14. https://dx.doi.org/10.14334/jitv.v24i1.1889

Tuiskula-Haavisto M, de Koning D J, Honkatukia M, Schulman N F, Maki-Tanila A, Vilkki J. 2004. Quantitative trait loci with parent-of-origin effects in chicken. Genet Res. 84(1): 57-66. https://doi.org/10.1017/s0016672304006950

Utama I V, Perdamaian A B I, Daryono B S. 2018. Plumage uniformity, growth rate and growth hormone polymorphism in indonesian hybrid chickens. Int J Poult Sci. 17(10): 486-492.

Wang H, Cahaner A, Lou L, Zhang L, Ge Y, et al. 2021. Genetics and breeding of a black-bone and blue eggshell chicken line. 1. Body weight, skin color, and their combined selection. Poultry Science. 100: 101035. https://doi.org/10.1016/j.psj.2021.101035

Wang J, Yuan X, Ye S, Huang S, He Y, et al. 2019. Genome wide association study on feed conversion ratio using imputed sequence data in chickens. Asian-Australas J Anim Sci. 32(4): 494-500. https://doi.org/10.5713/ajas.18.0319

Xu H, Zeng H, Luo C, Zhang D, Wang Q, Sun L, Yang L, Zhou M, Nie Q, Zhang X. 2011. Genetic effects of polymorphisms in candidate genes and the QTL region on chicken age at first egg. BMC Genetics. 12: 33. https://doi.org/10.1186/1471-2156-12-33

Yuan J, Sun C, Dou T, Yi G, Qu L, et al. 2015. Identification of promising mutants associated with egg production traits revealed by genome-wide association study. PLoS ONE. 10(10): e0140615. https://doi.org/10.1371/journal.pone.0140615

Zhang H, Zhang Y D, Wang S Z, Liu X F, Zhang Q, et al. 2010. Detection and fine mapping of quantitative trait loci for bone traits on chicken chromosome one. J Anim Breed Genet. 127: 462-468. https://doi.org/10.1111/j.1439-0388.2010.00871.x 
Zhou H, Deeb N, Evock-Clover C M, Ashwell, C M, Lamont S J. 2006. Genome-wide linkage analysis to identify chromosomal regions affecting phenotypic traits in the chicken. i. growth and average daily gain. Poultry Science. 85(10): 1700-1711. https://doi.org/10.1093/ps/85.10.1700

Zhou H, Deeb N, Evock-Clover C M, Mitchel A D, Ashwell C M, Lamont S J. 2007. Genome-wide linkage analysis to identify chromosomal regions affecting phenotypic traits in chicken. iii. skeletal integrity. Poultry Science. 86(2): 255-266. https://doi.org/10.1093/ps/86.2.255 


\section{Supplemental File 1. Parameters of MoBPSweb v.1.6.62}

Table 1. Phenotypes and major QTLs.

\begin{tabular}{|c|c|c|c|c|c|c|c|c|c|c|c|c|c|c|c|c|}
\hline \multirow[b]{2}{*}{ Traits } & \multirow[b]{2}{*}{ CHR } & \multirow[b]{2}{*}{ QTL peak/scan } & \multirow[b]{2}{*}{ QTL position } & \multirow[b]{2}{*}{$p$} & \multirow[b]{2}{*}{ PHENO. $\mu( \pm$ SD $)$} & \multicolumn{3}{|l|}{ Effect } & \multirow{2}{*}{$\begin{array}{l}\text { Allele } \\
\quad \text { frequency }\end{array}$} & \multirow{2}{*}{$\begin{array}{r}\text { Polygenic } \\
\text { loci } \\
\end{array}$} & \multirow[b]{2}{*}{$\mathrm{r}$} & \multirow[b]{2}{*}{$h^{2}( \pm \mathrm{SE})$} & \multirow[b]{2}{*}{ Maternal } & \multirow[b]{2}{*}{ Paternal } & \multirow{2}{*}{$\begin{array}{l}\text { Value } \\
(€)\end{array}$} & \multirow[b]{2}{*}{ Gene/SNP } \\
\hline & & & & & & AA & $\mathrm{AB}$ & $\mathrm{BB}$ & & & & & & & & \\
\hline $\mathrm{AFE}^{\mathrm{a}}$ & 1 & $207 \mathrm{cM} / 205-215 \mathrm{cM}$ & ${ }^{3} \mathrm{MCW} 0007 / /^{4} \mathrm{MCW} 0018$ & $<0.05$ & $159.95 \mathrm{~d}(1.1)$ & 1.74 & 0.87 & 0.39 & $\mathrm{~A}(0.53) / \mathrm{B}(0.47)$ & 1000 & 0.6 & $0.51(0.09)$ & 0.065 & 11.98 & 1.3 & MTNR1B/JQ249894 \\
\hline$B W 49 D^{b}$ & 1 & $205 / 205-241 \mathrm{cM}$ & MCW0018/MCW0058 & 0.0061 & 728.66 gr (16.3) & 42.66 & -8.66 & -34.66 & $\mathrm{~A}(0.57) / \mathrm{B}(0.43)$ & 1000 & 0.6 & $0.413(0.01)$ & 10 & 0 & 2 & $c G H / G+1705 A$ \\
\hline $\mathrm{BW} 56 \mathrm{D}^{\mathrm{c}}$ & 1 & $444 / 0-100 \mathrm{cM}$ & Unidentified & $<0.01$ & $858.84 \mathrm{gr}(158.84)$ & -23.8 & 58 & 11.41 & $\mathrm{~A}(0.43) / \mathrm{B}(0.57)$ & 1000 & 0.3 & 0.25 & 0.42 & 0 & 1 & $A T G L / c .782 G>A$ \\
\hline $\mathrm{EN}^{\mathrm{d}}$ & 1 & $145 / 122-205 \mathrm{cM}$ & ${ }^{3} \mathrm{ADL} 0019 /{ }^{4} \mathrm{ADL} 0150$ & $<0.05$ & 84.58 count $/ \mathrm{d}(6.07)$ & -14.22 & 40.34 & 30.56 & $\mathrm{~A}(0.41) / \mathrm{B}(0.59)$ & 1000 & 0.3 & $0.24(0.07)$ & 0 & 0 & 1.5 & $r s 14878430$ \\
\hline $\mathrm{FCR}^{\mathrm{e}}$ & 1 & $50 / 98-128 \mathrm{cM}$ & ${ }^{3} \mathrm{MCW} 0011 /{ }^{4} \mathrm{ADL} 0307$ & $<0.01$ & $1.82 \mathrm{~kg} / \mathrm{kg}(0.31)$ & 0.01 & 0.11 & 1.64 & $\mathrm{~A}(0.95) / \mathrm{B}(0.05)$ & 1000 & 0.1 & $0.07(0.01)$ & 0.52 & 0 & 2.3 & USP44 \\
\hline FEML $^{\mathrm{f}}$ & 1 & $72.6 / 72-94 \mathrm{cM}$ & ${ }^{2} \mathrm{MCW} 0010 /{ }^{5} \mathrm{MCW} 0106$ & & $10.64 \mathrm{~cm}(0.84)$ & 0.1 & 0.3 & 0.6 & $\mathrm{~A}(0.24) / \mathrm{B}(0.76)$ & 1000 & 0.95 & 0.92 & 0 & 1 & 1.05 & LRCHI \\
\hline $\mathrm{TL}^{\mathrm{g}} \mathrm{C}$ & 1 & $98 / 0-100 \mathrm{cM}$ & ${ }^{1} \mathrm{MCW} 0011$ & $<005$ & $1521 \mathrm{~cm}(0.84)$ & 011 & 0.53 & 19 & $\mathrm{~A}(023) / \mathrm{B}(0.77)$ & 1000 & 06 & 0.59 & 0 & 1 & 104 & $L C O R L / r s 314487178$ \\
\hline
\end{tabular}

${ }^{1}$ Peak/2Upper.suggest $/{ }^{3}$ Upper.sig/ $/ 4$ Lower.sig/ $/{ }^{5}$ Lower.suggest; AFE: Age at First Egg; BW49D: Body Weight 49 Days; BW56D: Body Weight 56 Days; EN: Egg Number; FCR: Feed Conversion Ratio; FEML: Femur Length; TL: Tibia Length. ${ }^{a}$ Tuiskula-Haavisto et al. (2004), Goto et al. (2011), dan Xu et al. (2011); ' Jennen et al. (2005) dan Nie et al. (2005); ' Zhou et al. (2006), Nie et al. (2010), dan Wang et al. (2021); ${ }^{\mathrm{d} H a n s e n}$ et al. (2005), Li et al. (2013), dan Yuan et al. (2015); ${ }^{e}$ De Koning et al. (2004) dan Wang et al. (2019); 'Zhang et al. (2010); ' Zhou et al. (2007).

\begin{tabular}{c|ccccccc}
\multicolumn{7}{|c}{ Table 2. Residual and genetic correlation. } \\
\hline & BW49D & AFE & BW56D & EN & FCR & FEML & TL \\
\hline BW49D & 1 & -0.75 & -0.5 & 0.25 & 0.5 & -0.9 & -0.9 \\
AFE & -0.5 & 1 & 0.75 & 0.14 & 0.9 & 0.2 & 0.2 \\
BW56D & -0.8 & 0.3 & 1 & 0.13 & 0.9 & 0.4 & 0.4 \\
EN & 0.8 & -0.2 & -0.4 & 1 & 0.6 & -0.4 & -0.43 \\
FCR & 0.95 & 0.85 & 0.95 & 0.6 & 1 & 0.1 & 0.2 \\
FEML & -0.5 & 0.2 & 0.32 & -0.21 & 0.54 & 1 & 0.6 \\
TL & -0.25 & 0.4 & 0.43 & -0.34 & 0.56 & 0.71 & 1 \\
\hline
\end{tabular}

Below the diagonal is genetic correlation; above the diagonal is residual correlation; values are generated via MoBPSweb server.

\begin{tabular}{|c|c|c|c|c|c|c|c|c|}
\hline Selection indexes & Standardization & BW49D & AFE & BW56D & EN & FCR & FEML & TL \\
\hline Broiler & Genomic $\mathrm{SD}^{1}$, Breeding Value $\mathrm{SD}^{2}$, Phenotypic SD & 4 & 2 & 4 & 2 & 5 & 2 & 2 \\
\hline Pelung & Genomic $\mathrm{SD}^{1}$, Breeding Value $\mathrm{SD}^{2}$, Phenotypic $\mathrm{SD}^{3}$ & 2 & 3 & 2 & 3 & 2 & 5 & 5 \\
\hline Phenotyping classes & Phenotyping cost & BW49D & AFE & BW56D & EN & FCR & FEML & TL \\
\hline Fully phenotyped & 50 & 50 & 50 & 50 & 50 & 50 & 50 & 50 \\
\hline
\end{tabular}

Fully phenotyped 50

${ }^{1}$ Scenario_1; ${ }^{2}$ Scenario_2; ${ }^{3}$ Scenario_3; SD: Standard Deviation; AFE: Age at First Egg; BW49D: Body Weight 49 Days; BW56D: Body Weight 56 Days; EN: Egg Number; FCR: Feed Conversion Ratio; FEML: Femur Length; TL: Tibia Length.

Table 4. General economy parameters.

Fixed cost ( $(€-\mathrm{Rp}) \quad$ Interest rate (\%) $\quad$ Genotyping cost per repeat $(€) \quad$ Number of Housing $(€) \quad$ Chicken feeds, vaccines, and supplements $(€)$

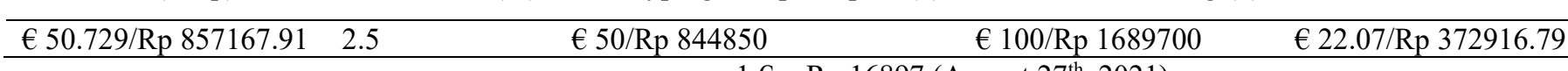




\section{Figures}

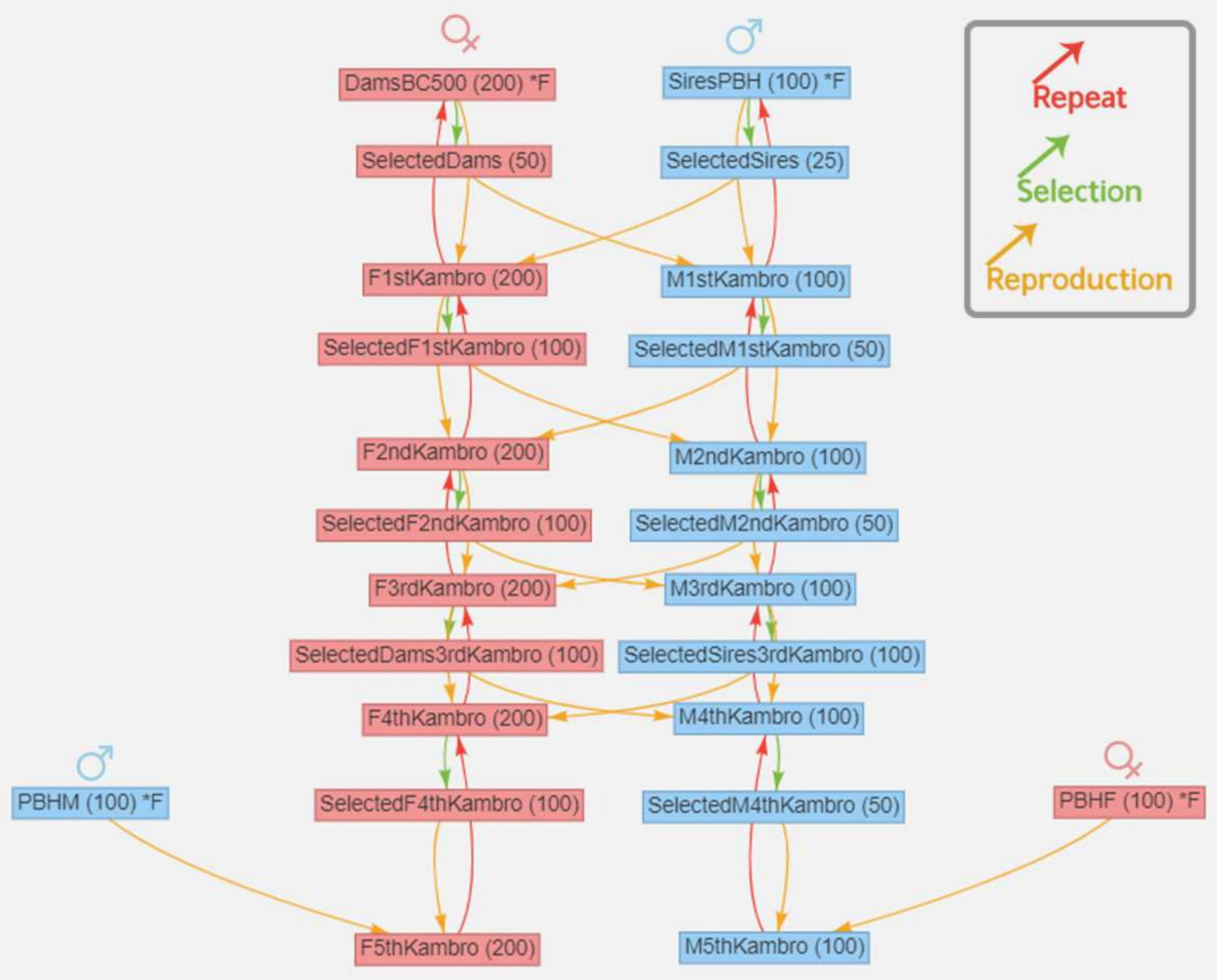

\section{Figure 1}

Gama Ayam Kambro breeding scheme. MoBPSweb (2021). 


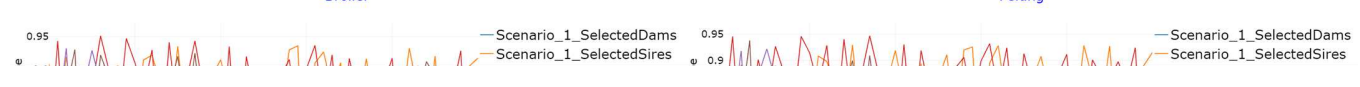

\section{Figure 2}

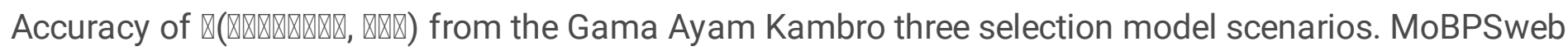
(2021). 
Average Inbreeding within Cohorts


Average Relationship within Cohorts
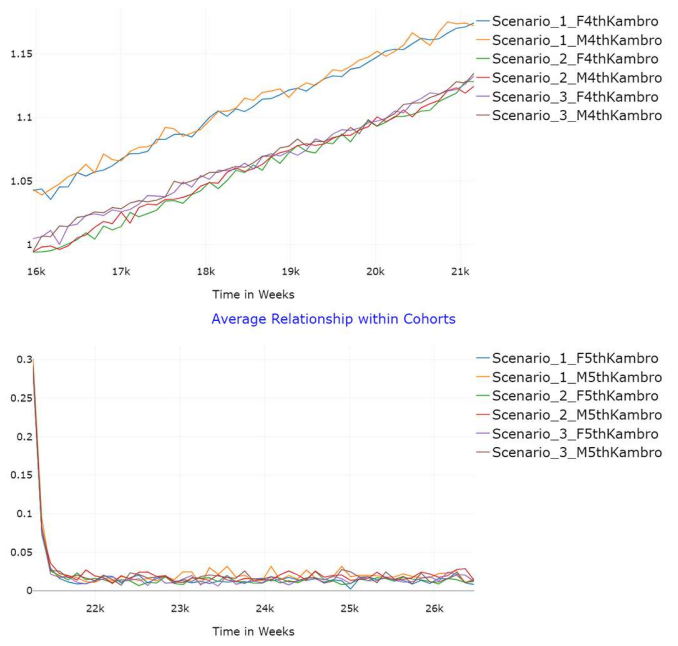

\section{Figure 3}

The F coefficient and weekly kinship of the Gama Ayam Kambro three selection model scenario. MoBPSweb (2021). 
AFE

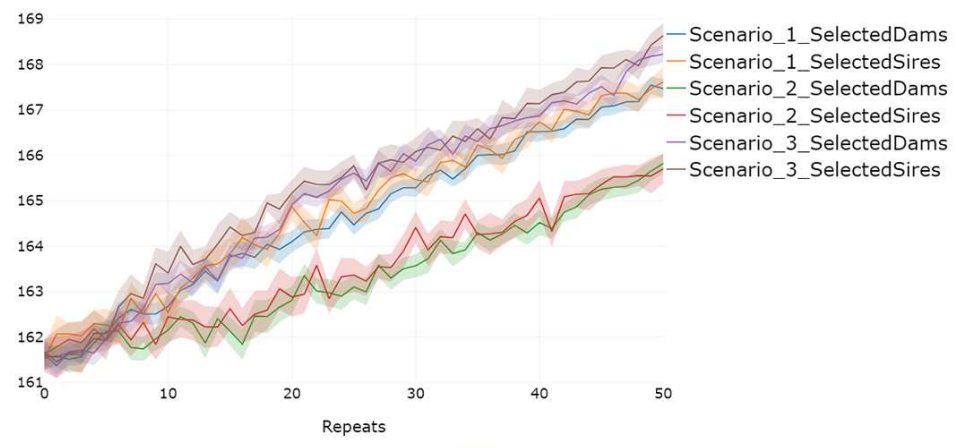

AFE

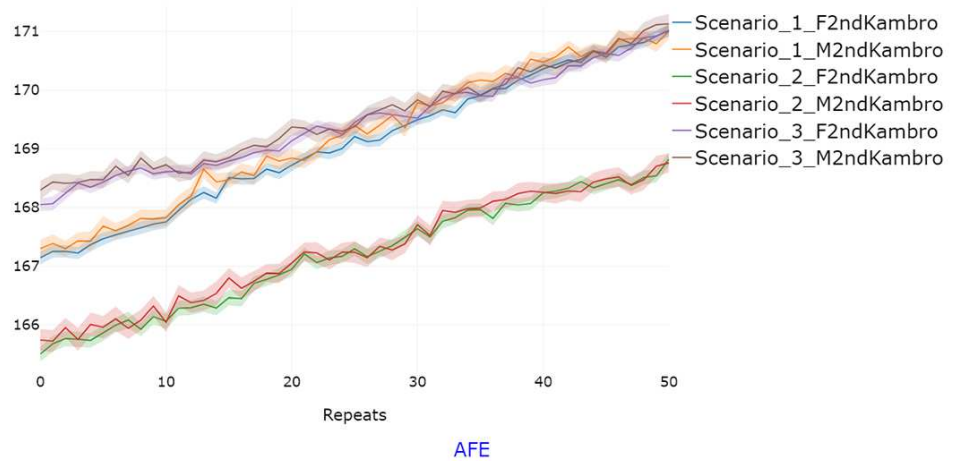

(1- F4thKambro

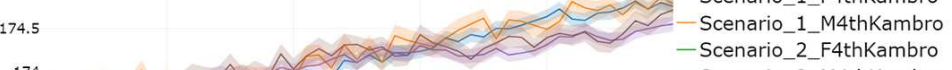

174 - Scenario_2_M4thKambro

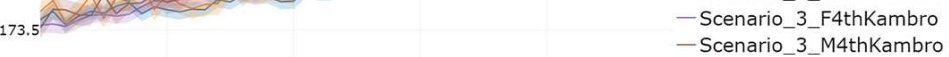

173

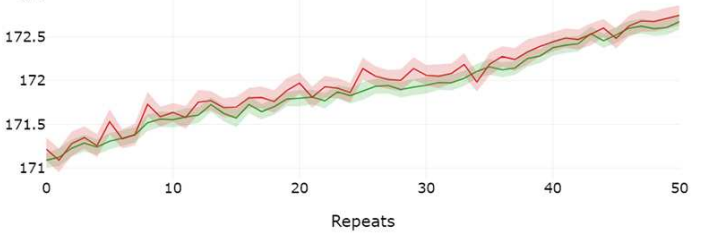

AFE

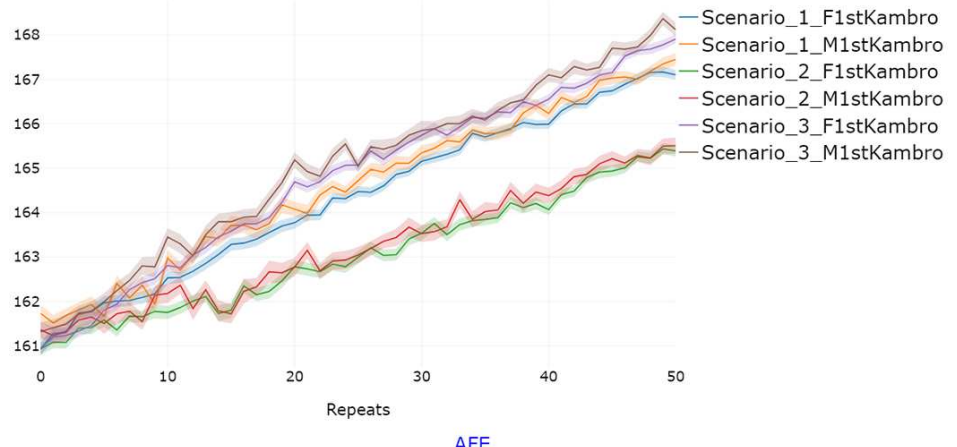

AFE
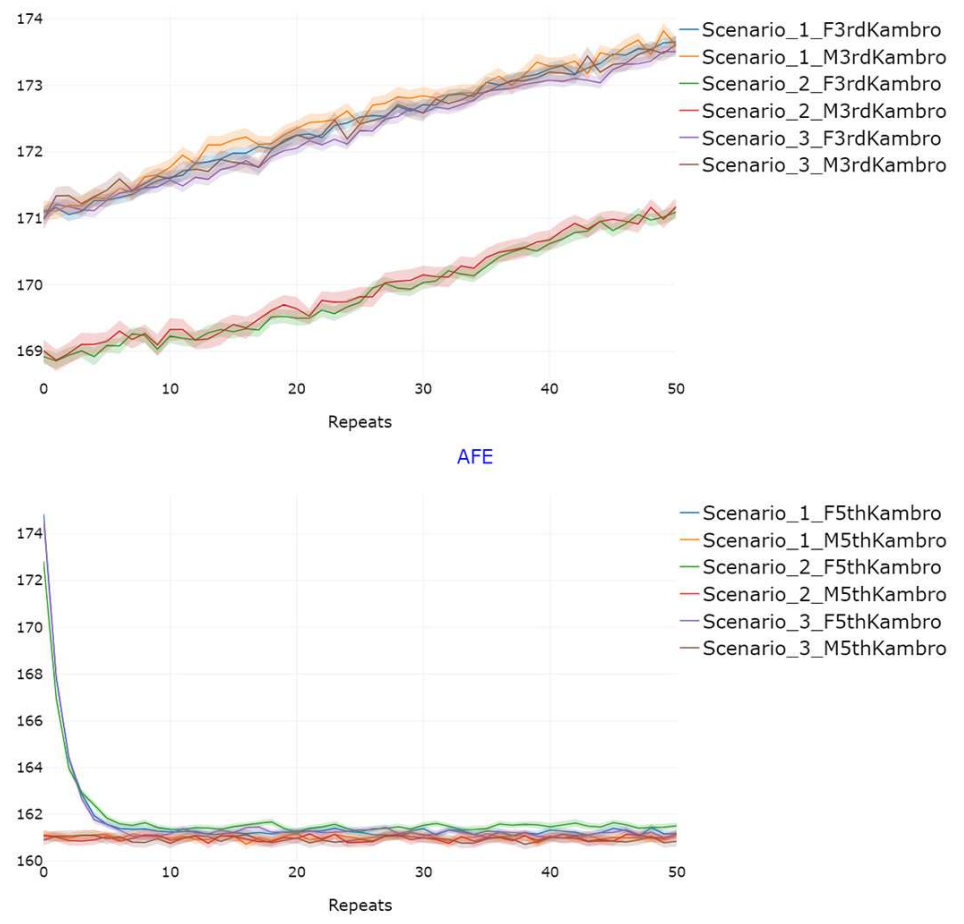

\section{Figure 4}

The observed phenotype of AFE from the Gama Ayam Kambro three selection model scenarios. MoBPSweb (2021). 
BW49D
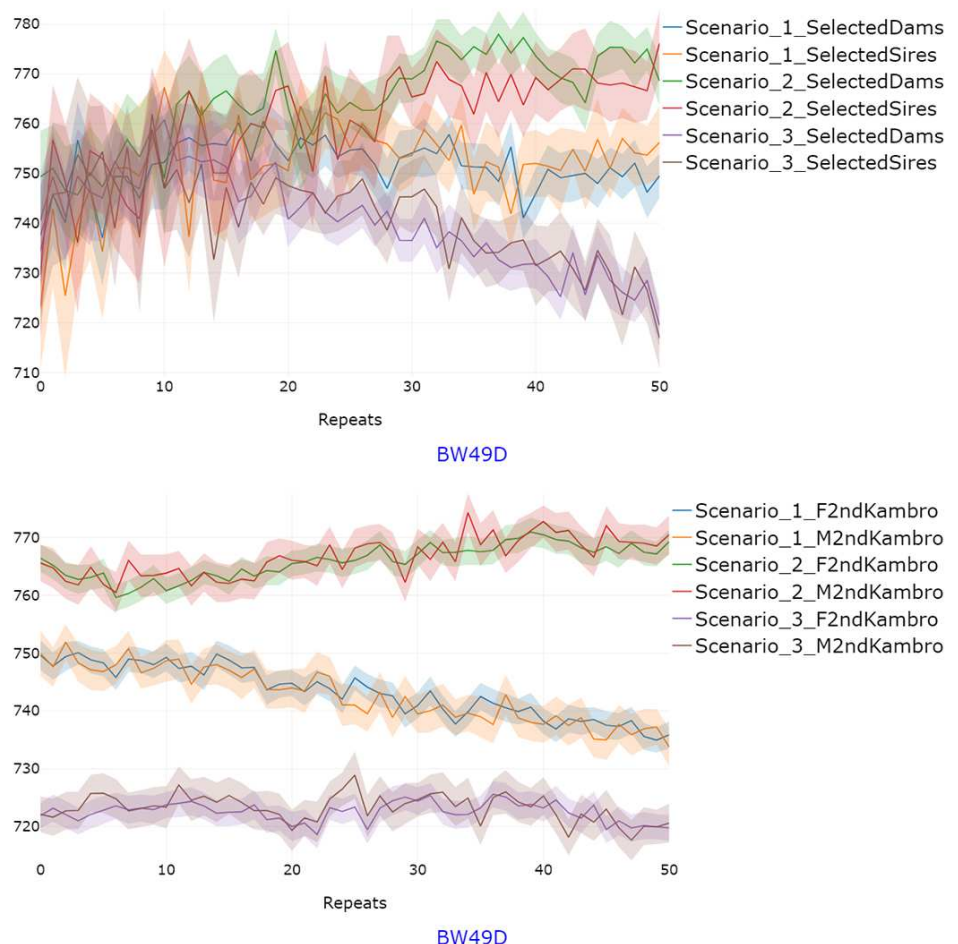

770-1) Scenario_1_F4thKambro

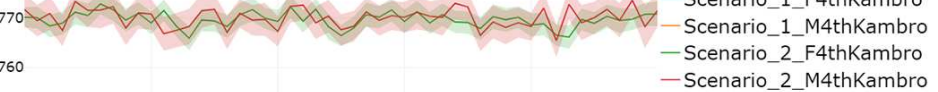

750

740

730

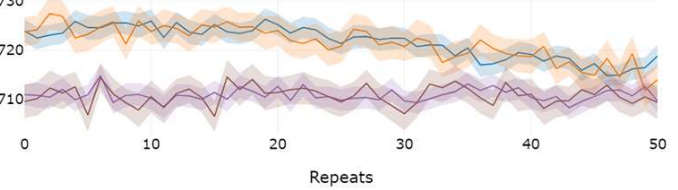

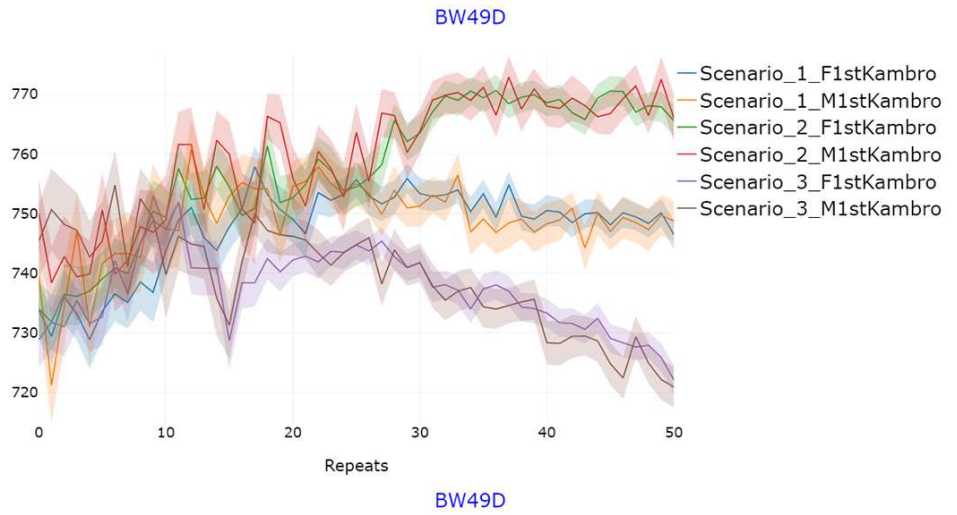

780 -Scenario_1_F3rdKambro 7700 - Scenario_1_F3rdKambro - Scenario_2_F3rdKambro - Scenario_3_F3rdKambro -Scenario_3_M3rdKambro 750

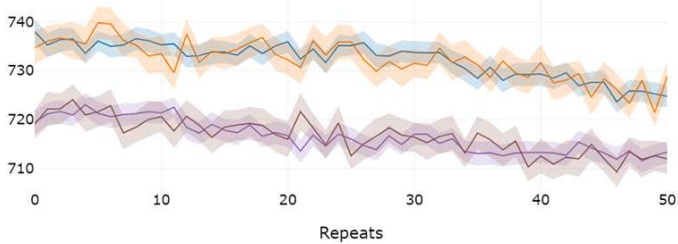

BW49D

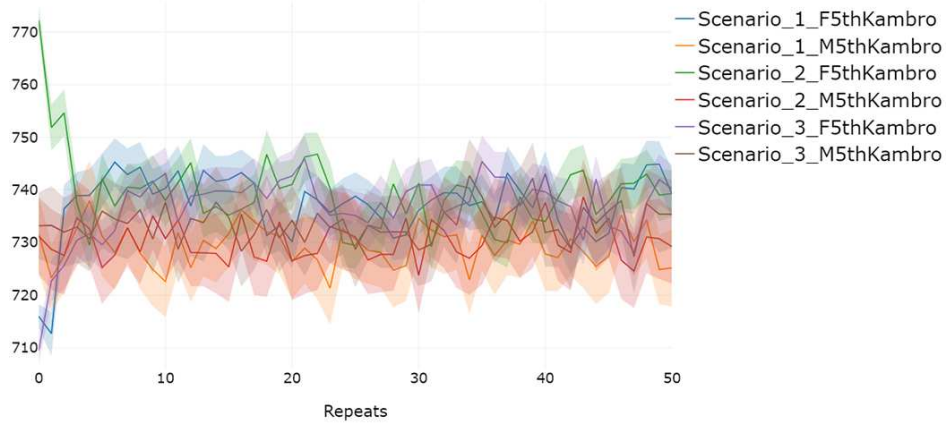

\section{Figure 5}

The observed phenotype of BW49D from the Gama Ayam Kambro three selection model scenarios. MoBPSweb (2021). 
BW56D

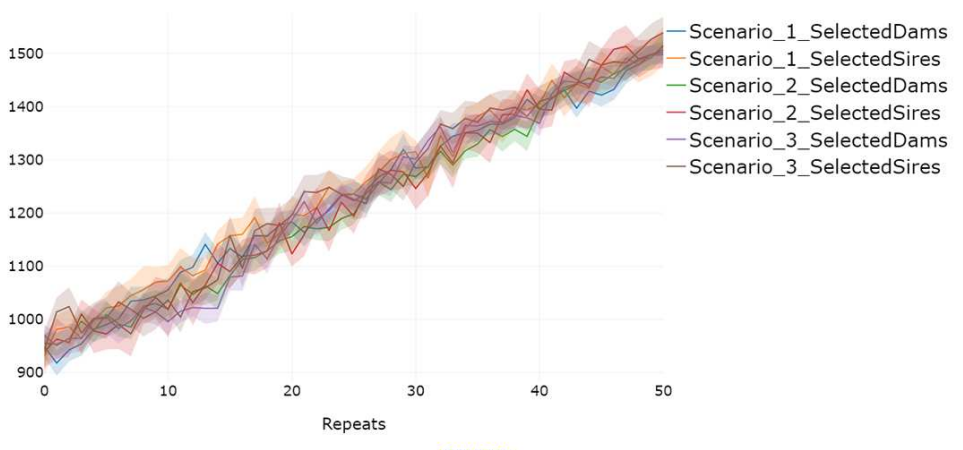

BW56D
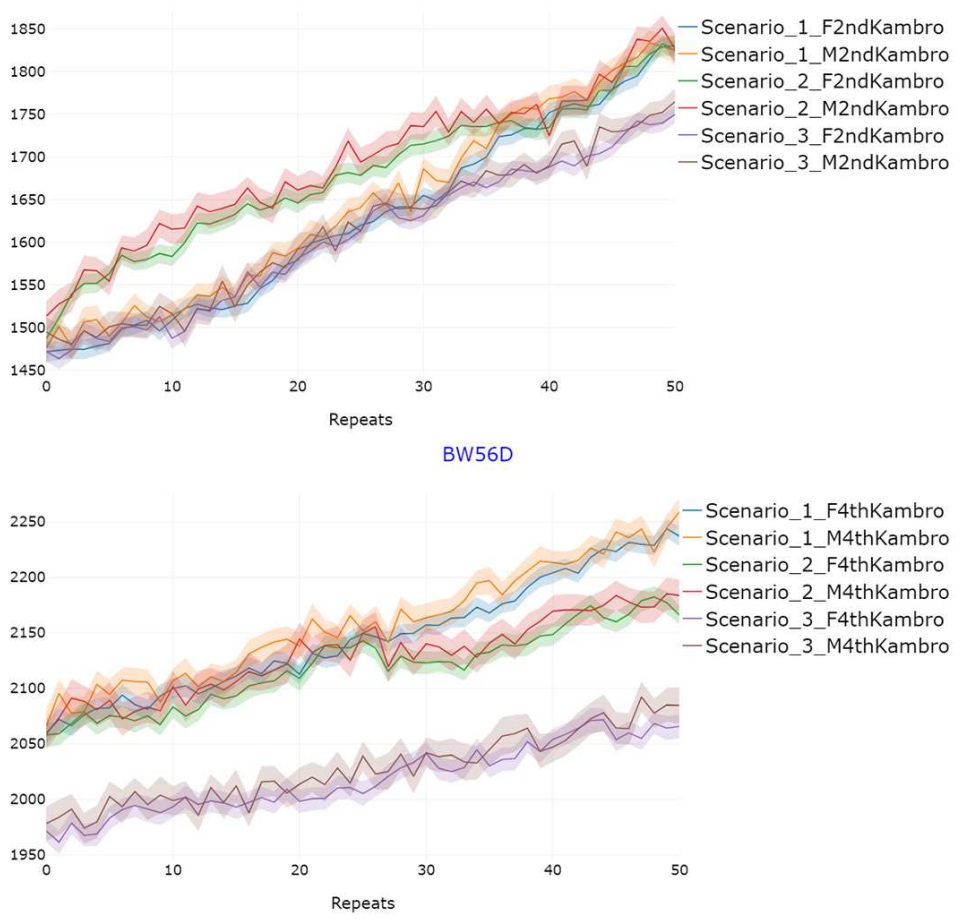

BW56D
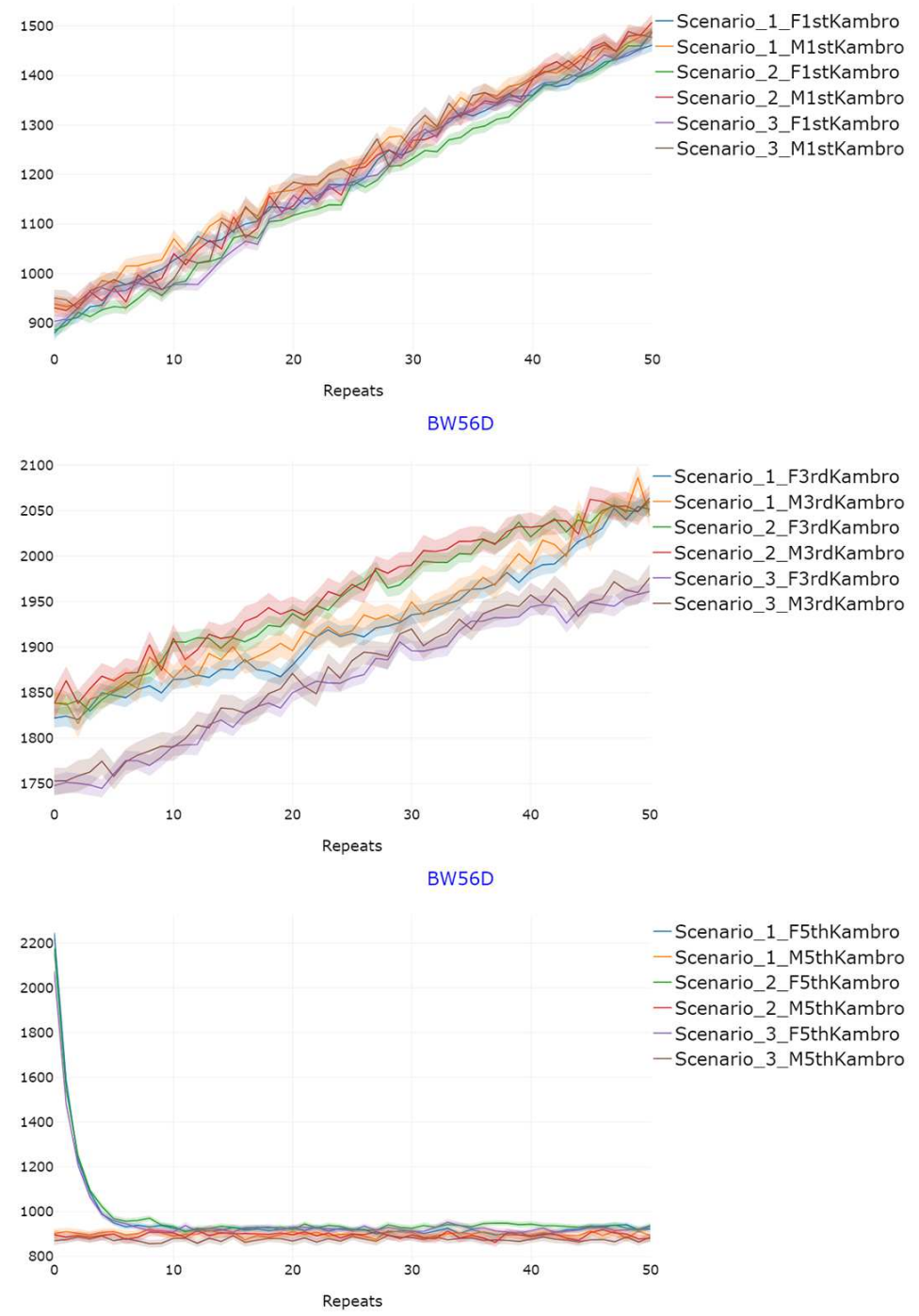

-Scenario_1_F5thKambro - Scenario_1_M5thKambro -Scenario_2_F5thKambro

-Scenario_2_M5thKambro

- Scenario_3_F5thKambro

- Scenario_3_M5thKambro

\section{Figure 6}

The observed phenotype of BW56D from the Gama Ayam Kambro three selection model scenarios. MoBPSweb (2021). 
EN

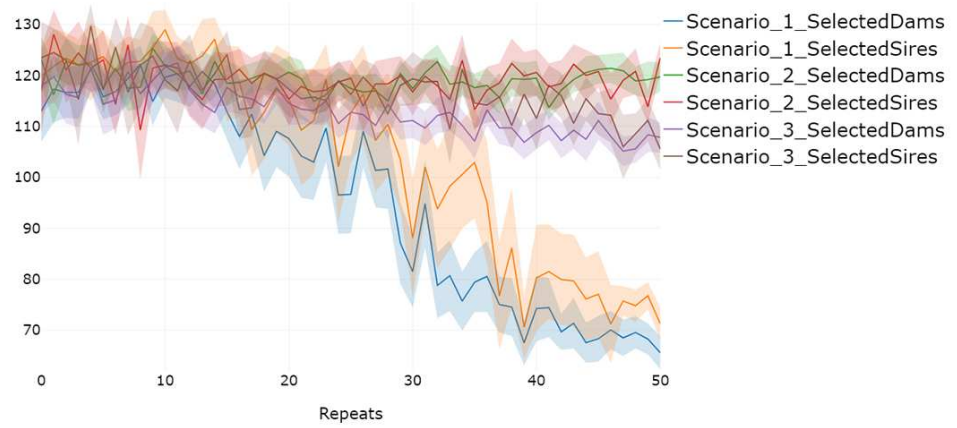

EN



90

80

70

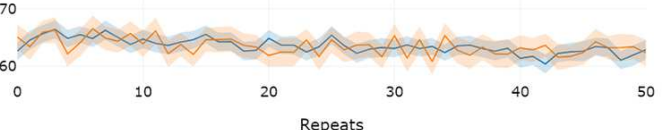

EN

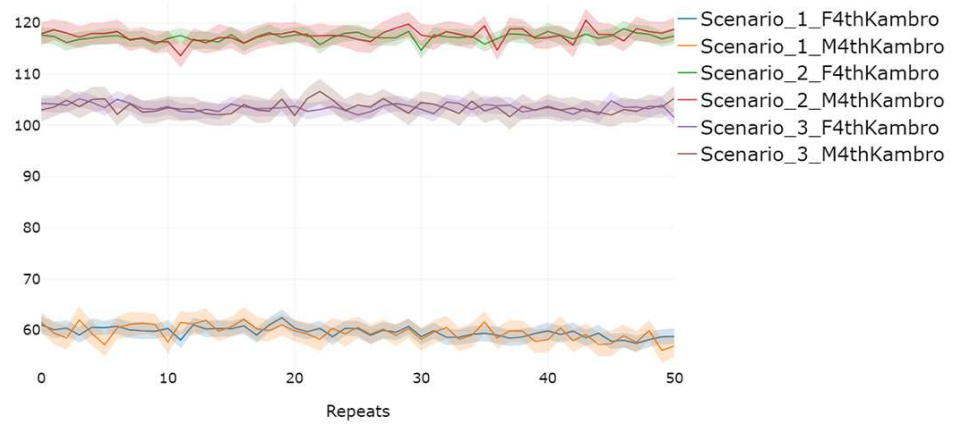

EN

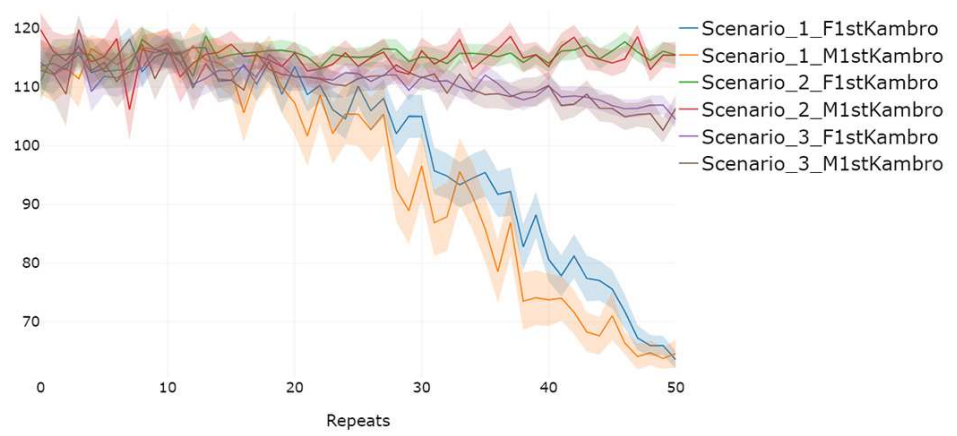

EN

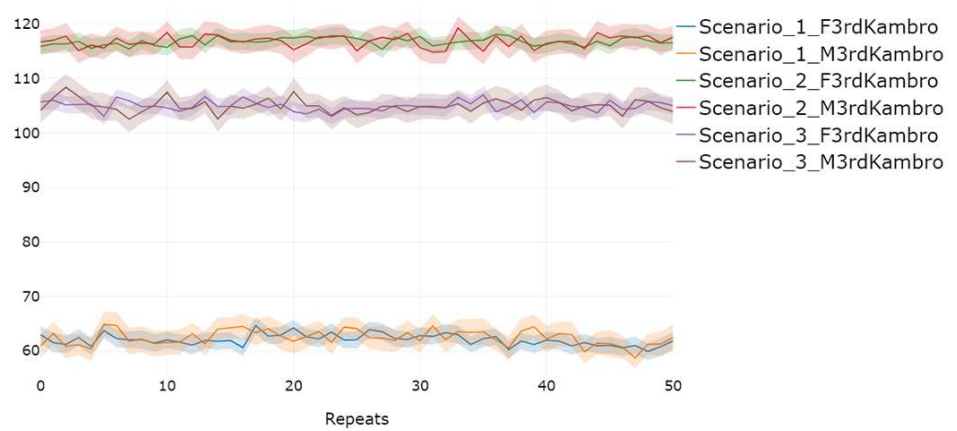

EN

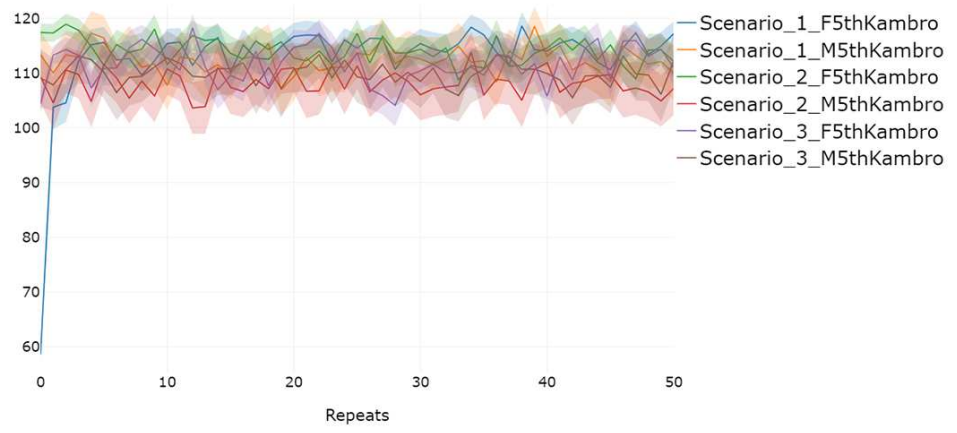

\section{Figure 7}

The observed phenotype of EN from the Gama Ayam Kambro three selection model scenarios. MoBPSweb (2021). 
FCR

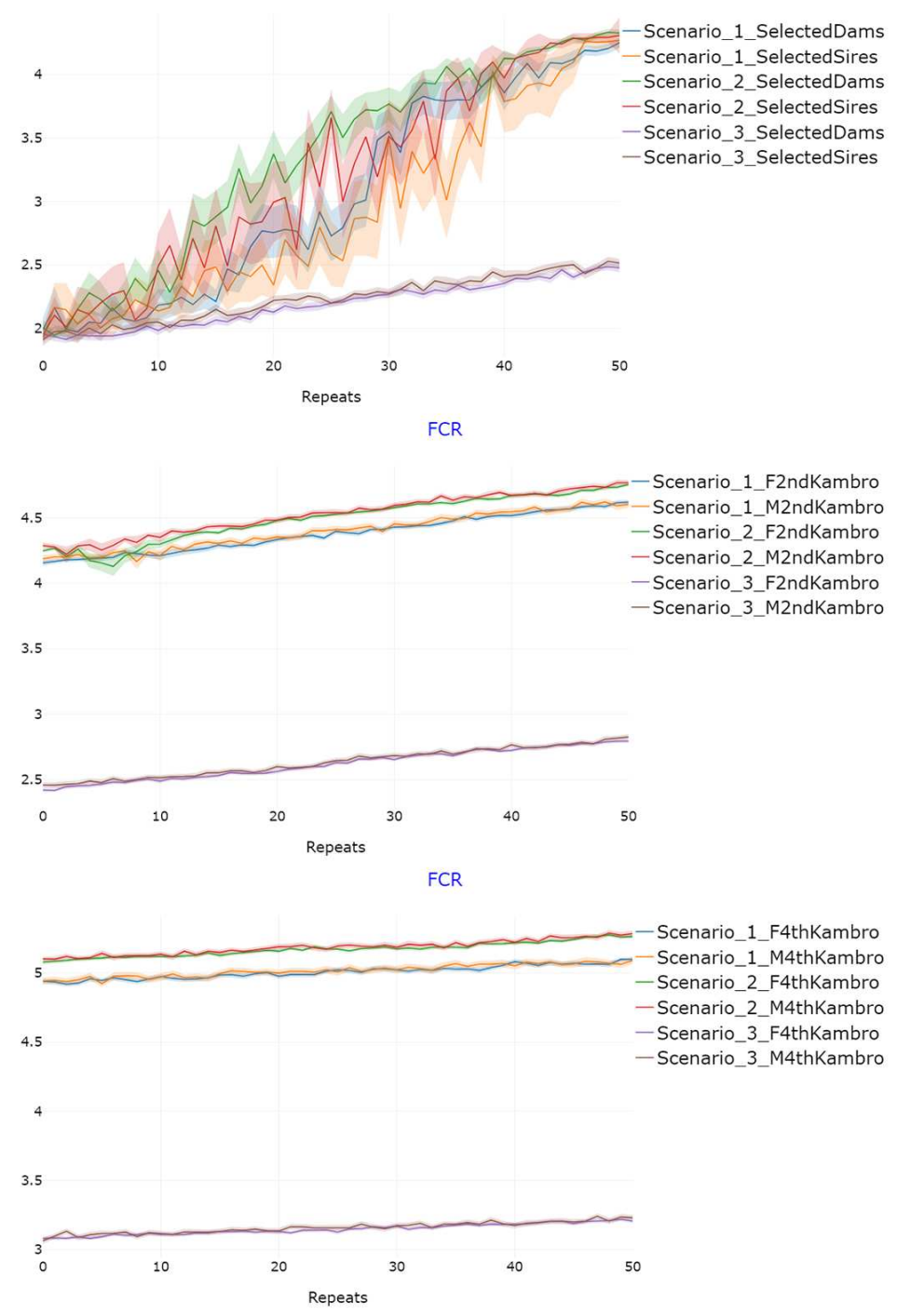

FCR
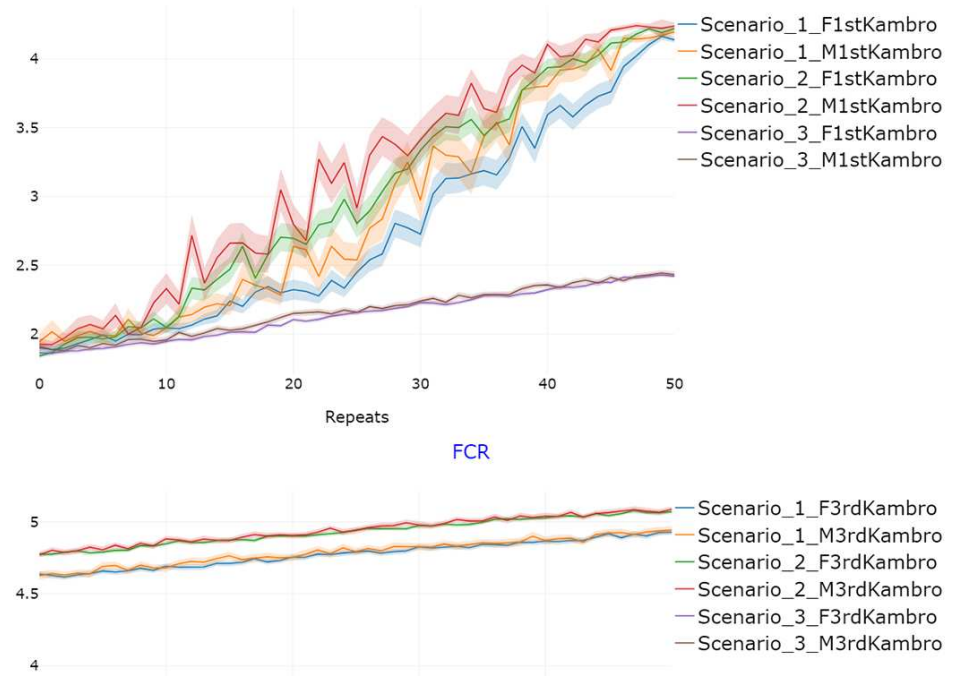

3.5
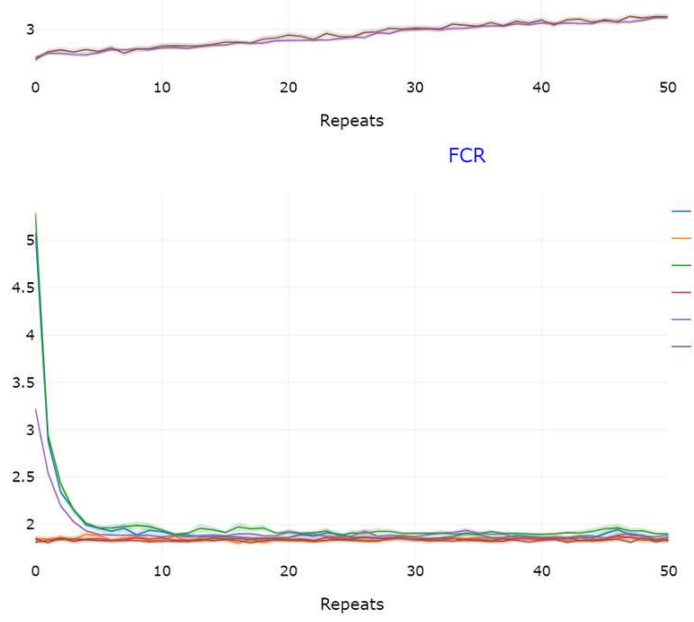

Scenario_1_F5thKambro Scenario_1_M5thKambro Scenario_2_F5thKambro

- Scenario_2 M5thKambro

- Scenario_3_F5thKambro

-Scenario_3_M5thKambro

\section{Figure 8}

The observed phenotype of FCR from the Gama Ayam Kambro three selection model scenarios. MoBPSweb (2021). 
FEML
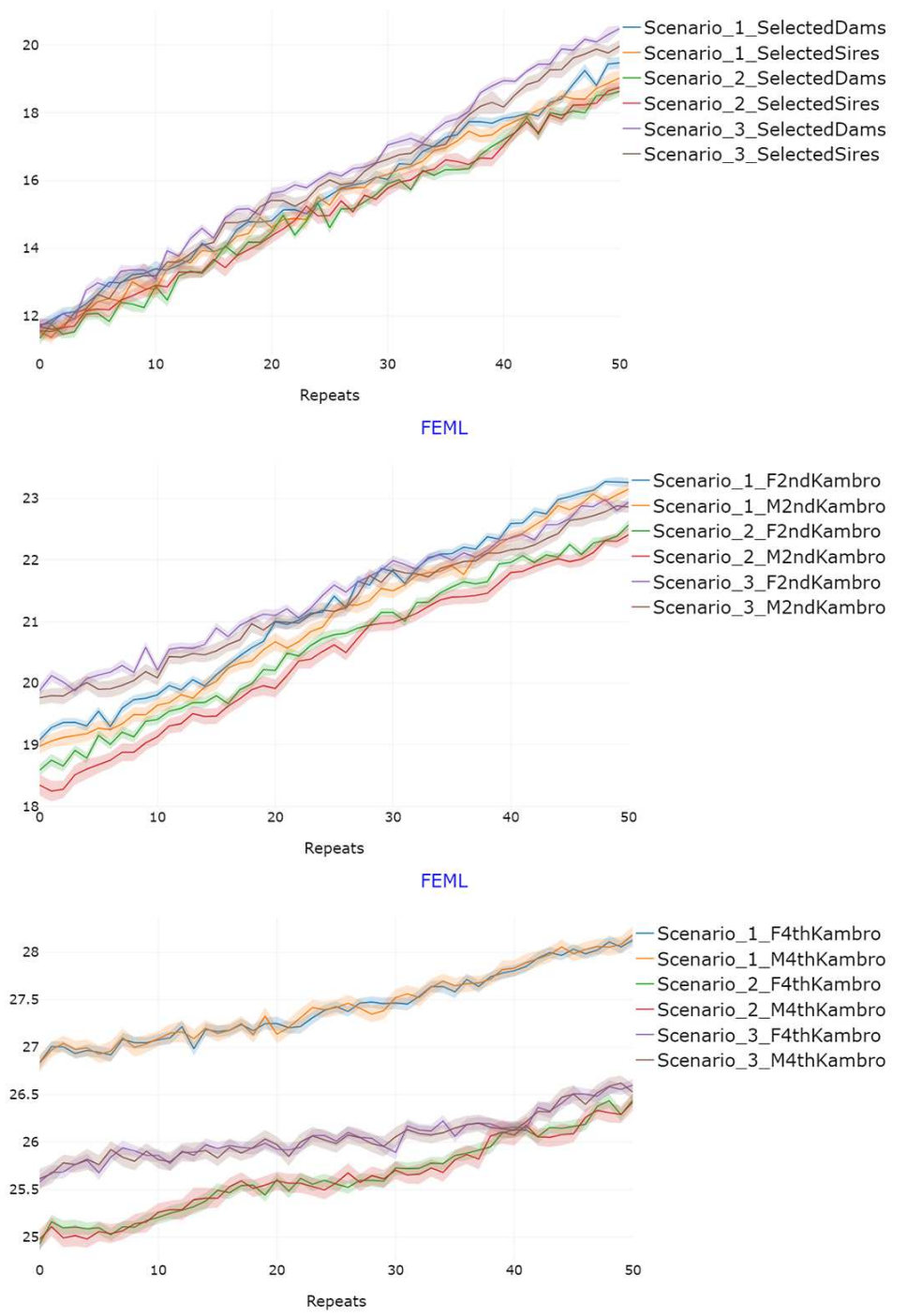

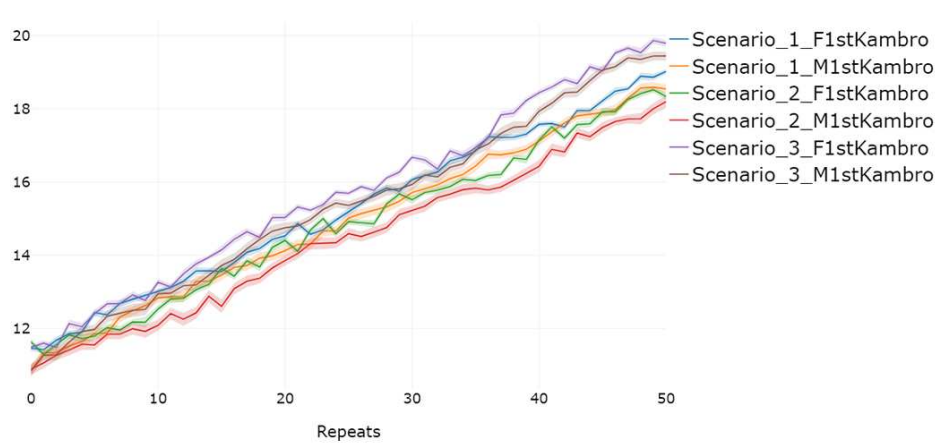

FEML

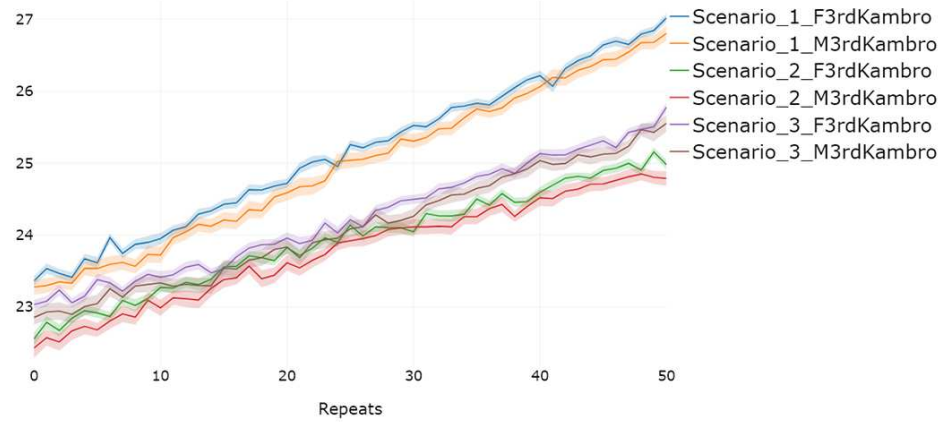

FEML



-Scenario_1_F5thKambro - Scenario_1_M5thKambro - Scenario_2_F5thKambro

- Scenario_2_M5thKambro

- Scenario_3_F5thKambro

- Scenario_3_M5thKambro

\section{Figure 9}

The observed phenotype of FEML from the Gama Ayam Kambro three selection model scenarios. MoBPSweb (2021). 
TL

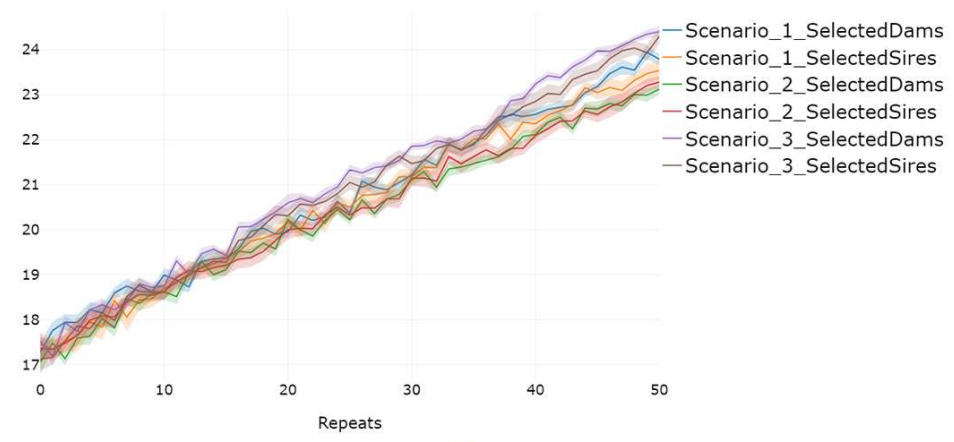

TL
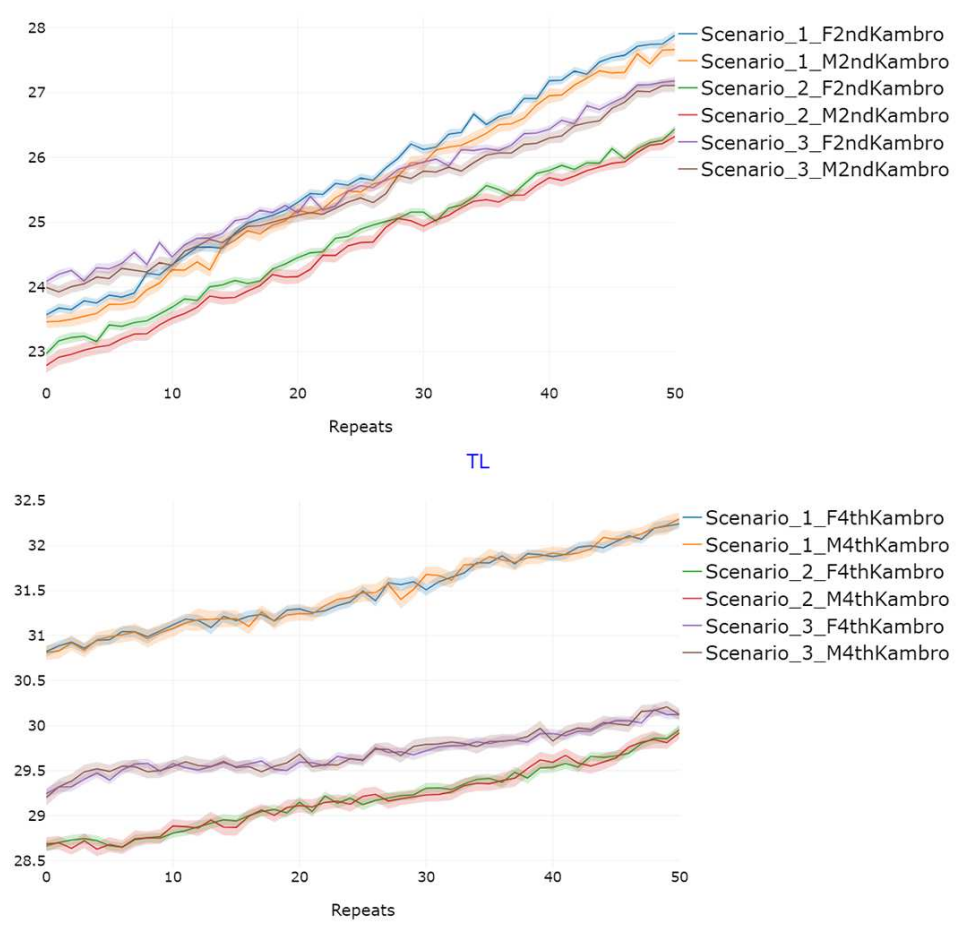

$\mathrm{TL}$

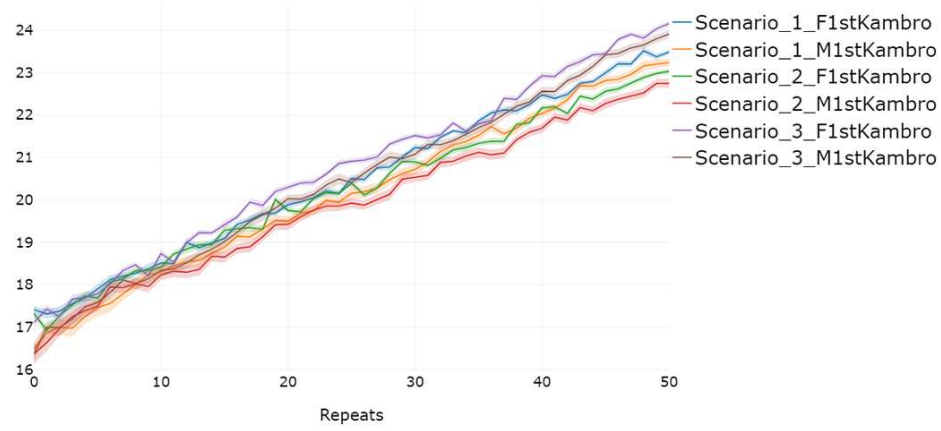

TL


\section{Figure 10}

The observed phenotype of TL from the Gama Ayam Kambro three selection model scenarios. MoBPSweb (2021).

\section{Supplementary Files}

This is a list of supplementary files associated with this preprint. Click to download.

- tbv08AFE.tif

- tbv08BW49D.tif

- tbv08BW56D.tif

- tbv08EN.tif 
- tbv08FCR.tif

- tbv08FEML.tif

- tbv08TL.tif

- SupplementalFileSIM08.pdf

- swarautamacosts.tif 